3 Natalia Gebara ${ }^{1}$, Julia Scheel ${ }^{2}$, Renata Skovronova ${ }^{1}$, Cristina Grange ${ }^{3}$, Luca Marozio ${ }^{4}$,

4 Shailendra Gupta ${ }^{2}$, Veronica Giorgione ${ }^{5}$, Federico Caicci $^{6}$, Chiara Benedetto ${ }^{4}$, Asma Khalil ${ }^{5,7}$,

$5 \quad$ Benedetta Bussolati ${ }^{1 *}$

6 1. Department of Molecular Biotechnology and Health Sciences, University of Turin, Italy.

7 2. Department of Systems Biology and Bioinformatics, University of Rostock, Germany.

8 3. Department of Medical Sciences, University of Turin, Turin, Italy.

9 4. Department of Surgical Sciences, Obstetrics and Gynecology, University of Turin, Italy.

\section{Single extracellular vesicle analysis in human amniotic fluid shows evidence of} phenotype alterations in preeclampsia

5. Vascular Biology Research Centre, Molecular and Clinical Sciences Research Institute, St George's University of London, UK

6. DiBio Imaging facility, University of Padua, Italy.

7. Fetal Medicine Unit, St George's University Hospitals NHS Foundation Trust, St George's University of London, UK.

*Corresponding Author: Benedetta Bussolati, Molecular Biotechnology Center, University of Turin, via Nizza 52, 10126 Turin, Italy. Fax 011-6331184, Tel. 011-6706453, e-mail: benedetta.bussolati@unito.it

Running Title: Amniotic fluid-derived EVs

Disclosure: The authors declare no conflict of interest.

Funding: NG, JS and VG are part of the iPLACENTA and RS of RenalToolBox projects, which have received funding from the European Union's Horizon 2020 research and innovation program under the Marie Skłodowska-Curie grant agreement No. 765274 and No. 813839 respectively. 


\section{Abstract}

28 Amniotic fluid surrounding the developing fetus is a complex biological fluid rich in metabolically active bio-factors. The presence of extracellular vesicles (EVs) in amniotic fluid has been mainly related to fetal urine. We here characterized EVs from term amniotic fluid in terms of surface marker expression using different orthogonal techniques. EVs appeared to be a heterogeneous population expressing markers of renal, placental, epithelial and stem cells. Moreover, we compared amniotic fluid EVs from normal pregnancies with those of preeclampsia, a hypertensive disorder affecting up to $8 \%$ of pregnancies worldwide. An increase of CD105 (endoglin) expressing EVs was observed in preeclamptic amniotic fluid by bead-based cytofluorimetric analysis, and further confirmed using a chip-based analysis. HLA$\mathrm{G}$, a typical placental marker, was not co-expressed by the majority of $\mathrm{CD} 105^{+} \mathrm{EVs}$, suggesting their origin from amniotic fluid cells. At a functional level, preeclampsia-derived EVs, but not normal pregnancy EVs, showed an antiangiogenic effect, possibly due to the decoy effect of endoglin. In addition, several miRNAs were differentially expressed in preeclampsia-derived EVs and directly related to the modulation of angiogenesis and trophoblast function. Our results provide a characterization of term amniotic fluid-EVs, supporting their origin from fetal and placental cells. In preeclampsia, the observed antiangiogenic characteristics of amniotic fluid-EVs may reflect the hypoxic and antiangiogenic microenvironment and could possibly impact on the developing fetus or on the surrounding fetal membranes.

47 Key-words: extracellular vesicles, exosomes, amniotic fluid, placenta, pregnancy disorders, angiogenesis, soluble endoglin. 


\section{Introduction}

Amniotic fluid is a complex biological fluid surrounding the developing fetus and in close proximity to the placenta (Underwood, Gilbert and Sherman, 2005). Its composition, after fetal skin keratinization, mainly consists of fetal cardiovascular, renal, pulmonary, and endothelial secretions (Roubelakis, Trohatou and Anagnou, 2012). Amniotic fluid has a relevant role in fetal physiology; it not only acts as a protective cushion to mechanical injury but also contains a plethora of active factors, including nutrients, growth and antimicrobial factors, cells and extracellular vesicles (EVs) (Underwood, Gilbert and Sherman, 2005).

EVs are a highly heterogeneous population of small particles enclosed in a lipid bilayer, released by almost all cell types, and involved in cell-to-cell signalling. In pregnancy, EVs are essential for diverse physiological and pathological processes (Jankovičová et al., 2020) and may play a role in angiogenesis and successful fetal development (Gebara et al., 2021). The role of EVs derived from placental trophoblasts has been studied extensively, as they are continuously released into the maternal bloodstream and play a crucial role in regulating the maternal immune response and pregnancy adaptation (Nair and Salomon, 2018). At variance, the profile and function of EVs present in amniotic fluid have been poorly investigated, as their characteristics have been mainly attributed to fetal urine production (Keller et al., 2007). Indeed, previous studies showed that a large proportion of EVs within the amniotic fluid express typical renal markers, such as aquaporin-2 and CD24, demonstrating their fetal urine origin (Keller et al., 2007). However, multiple cells may release EVs into the amniotic fluid, including the amnion, placental tissues and fetal cells (Dixon et al., 2018; McMaster et al., 1998). Interestingly, isolated amniotic epithelial or stem cells in culture were shown to release EVs with trophic, immunomodulating and anti-inflammatory functions (Balbi et al., 2017; Sedrakyan et al., 2017). It is therefore conceivable that EVs released from those cells are also 
pharmaceutical product, including term amniotic fluid-EVs, against the COVID-19 related cytokine storm (Mitrani et al., 2021). The analysis and characterization of amniotic fluid EVs is therefore of interest.

Preeclampsia is a complex pregnancy disorder characterized by new-onset hypertension with associated maternal organ dysfunction, including impaired liver function, renal damage (significant proteinuria, oligoanuria, increased creatinine), low platelets, haemolysis, decreased $\mathrm{sPO}_{2}$ or pulmonary edema, or visual and cerebral disturbances (Brown et al., 2018). Preeclampsia affects up to $8 \%$ of pregnancies world-wide (Duley, 2009) and causes significant maternal and perinatal morbidity and mortality, as well as long-term complications (Amaral et al., 2015). Abnormal placentation and insufficient trophoblast invasion are considered the main causes of preeclampsia (Kaufmann, Black and Huppertz, 2003), leading to the release of antiangiogenic factors that subsequently promote maternal endothelial dysfunction (Staff et al., 2007). Several studies highlighted the presence of altered EVs in the maternal circulation of preeclamptic women and their possible contribution to endothelial dysfunction (Escudero et al., 2016). Interestingly, EVs carrying antiangiogenic factors, such as soluble vascular endothelial growth factor receptor 1 (sFlt-1) and soluble endoglin, were found at high levels in the maternal circulation and were shown to be released by placental explants (Tannetta et al., 2013; Chang et al., 2018; Nair et al., 2021). Therefore, it is of interest to evaluate whether amniotic fluid EVs in preeclamptic pregnancies might have phenotypic or functional differences with respect to those of normal pregnancies.

In the present study, we aimed to characterize amniotic fluid-derived EVs from term normal and preeclamptic pregnancies. Surface marker expression was analyzed at a single-EV level using different techniques, such as super-resolution microscopy, ExoView array and bead- 
based cytofluorimetric analysis. In addition, their differential microRNA (miRNA) profile and angiogenic properties were investigated.

\section{Materials and methods}

102 Collection of amniotic fluid: Amniotic fluid samples (40 from normal pregnancies and 7 from

103 preeclamptic pregnancies) were collected during caesarean delivery in the Department of

104 Surgical Sciences at the University of Turin, after approval by the Ethics Review Board of the

105 Health and Science City of Turin, (Città della Salute e della Scienza di Torino, protocol N ${ }^{\circ}$ provided preoperative written informed consent. The fluid was collected by sterile acupuncture after myometrial incision, before the incision of the amniotic membranes. Three of the preeclamptic patients $(\mathrm{n}=7)$ were affected by comorbidities, including fetal growth restriction,

111 type two diabetes, and systemic lupus erythematosus. Further clinical information about 112 preeclamptic patients can be found in Table 2.

113 Table 1. Clinical information showing the mean $\pm S D$ of gestational age, maternal age at 114 delivery and the volume of the amniotic fluid obtained from normal and preeclamptic 115 pregnancies.

\begin{tabular}{lcc}
\hline & $\begin{array}{c}\text { Normotensive pregnancies } \\
(\mathbf{n}=\mathbf{4 0})\end{array}$ & $\begin{array}{c}\text { Preeclampsia } \\
(\mathbf{n}=\mathbf{7})\end{array}$ \\
\hline Gestational age (weeks) & $37.9 \pm 1.8$ & $36.1 \pm 2.6$ \\
\hline Maternal age (years) & $35.7 \pm 5.2$ & $32.8 \pm 6.7$ \\
\hline Amniotic fluid volume (m) & $19.9 \pm 7.8$ & $18.4 \pm 11.3$ \\
\hline
\end{tabular}


119 Table 2. Clinical information on individual preeclamptic patients showing the gestational age,

120 maternal age at delivery, week of preeclampsia diagnosis, sFlt-1/PIGF ratio, other

121 pathologies, treatment, and additional relevant information. PIGF:Placental growth factor.

\begin{tabular}{|c|c|c|c|c|c|c|c|}
\hline & $\begin{array}{c}\text { Age of } \\
\text { gestation } \\
\text { (weeks+days) }\end{array}$ & $\begin{array}{c}\text { Maternal } \\
\text { age } \\
\text { (years) }\end{array}$ & $\begin{array}{c}\text { Preeclampsia } \\
\text { diagnosis } \\
\text { (weeks) }\end{array}$ & $\begin{array}{l}\text { sFlt- } \\
\text { 1/PIGF } \\
\text { ratio }\end{array}$ & $\begin{array}{c}\text { Other } \\
\text { pathologies }\end{array}$ & Treatments & Other \\
\hline 1 & $36+1$ & 42 & 36 & 280 & - & $\begin{array}{l}\text { Betamethasone } \\
\text { Nifedipine }\end{array}$ & $\begin{array}{c}\text { Assisted } \\
\text { reproduction } \\
\text { techniques }\end{array}$ \\
\hline 2 & $32+1$ & 32 & 31 & 531 & - & $\begin{array}{c}\text { Betamethasone } \\
\text { Nifedipine } \\
\mathrm{MgSO}_{4}\end{array}$ & $\begin{array}{l}\text { Fetal growth } \\
\text { restriction }\end{array}$ \\
\hline 3 & $39+2$ & 22 & 36 & 42 & - & - & - \\
\hline 4 & $36+6$ & 39 & 35 & - & $\begin{array}{l}\text { Chronic } \\
\text { hypertension, } \\
\text { systemic } \\
\text { lupus } \\
\text { erythematosus }\end{array}$ & $\begin{array}{c}\text { Methyldopa, } \\
\text { Nifedipine, } \\
\text { Hydroxychloroquine } \\
\text {, Aspirin, Low } \\
\text { Molecular Weight } \\
\text { Heparin }\end{array}$ & - \\
\hline 5 & $38+2$ & 28 & 36 & - & $\begin{array}{c}\text { Type } 2 \\
\text { diabetes }\end{array}$ & Metformin, Insulin & - \\
\hline 6 & $36+1$ & 35 & 36 & - & - & $\begin{array}{l}\text { Labetalol, } \\
\text { Nifedipine }\end{array}$ & $\begin{array}{l}\text { Fetal growth } \\
\text { restriction }\end{array}$ \\
\hline 7 & $36+2$ & 33 & 33 & - & - & $\begin{array}{c}\text { Labetalol, } \\
\text { Nifedipine, } \\
\text { Metformin, Insulin }\end{array}$ & - \\
\hline
\end{tabular}

122

123 Isolation and culture of term amniotic fluid mesenchymal stromal cells (AFSCs): Term

124 AFSCs were previously obtained and characterized, as described (Iampietro et al., 2020).

125 Briefly, amniotic fluid was transferred into a sterile $50 \mathrm{~mL}$ falcon and centrifuged at $500 \mathrm{~g}$ for

12610 minutes at room temperature. The cell pellet was resuspended in $\alpha$-MEM Medium

127 (Gibco/BRR, ThermoFisher, MA, USA) supplemented with 20\% Chang Medium B (Irvine

128 Scientific, Santa Ana, California, USA) and 2\% Chang Medium C (Irvine Scientific), 20\%

129 Fetal Calf Serum (Invitrogen, Carlsbad, CA, USA), $50 \mathrm{IU} / \mathrm{mL}$ penicillin, $50 \mathrm{~g} / \mathrm{mL}$

130 streptomycin, 5mM glutamine (all from Sigma-Aldrich, St. Louis, MO, USA). The cells were

131 placed into a T25 flask, incubated at $37^{\circ} \mathrm{C}$ on $5 \% \mathrm{CO}_{2}$ until the cells formed clusters (1-2

132 weeks). Afterwards, the medium was replaced twice a week until the cells reached confluency. 
133 Under these culture conditions, AFSCs expressed typical mesenchymal surface markers and

134 differentiation properties, as previously described (Iampietro et al., 2020).

135 EV Isolation from amniotic fluid: Amniotic fluid samples were diluted in PBS up to a volume

136 of $20 \mathrm{ml}$ and subjected to differential centrifugations. For the isolation of cells, samples

137 underwent a 5 min centrifugation at $300 \mathrm{x} \mathrm{g}$, at room temperature. A second centrifugation of

$13810 \mathrm{~min}$ was performed at $500 \mathrm{xg}$, at room temperature, for removal of debris. The supernatant

139 was then passed through a cell sieve and underwent a $30 \mathrm{~min}$ ultracentrifugation (70 Ti rotor,

140 Beckman Coulter, CA, USA) at $10,000 \mathrm{xg}$, at $4^{\circ} \mathrm{C}$. The resulting pellet was discarded, and the

141 supernatant was further centrifuged for $2 \mathrm{~h}$ at $100,000 \mathrm{x}$ g at $4^{\circ} \mathrm{C}$. The final step included

142 resuspension of EV pellets in $1 \mathrm{ml} \mathrm{1 \%} \mathrm{DMSO/PBS}$ and additional filtration through a $0.22 \mu \mathrm{m}$

143 filter. The recovered EVs were stored at $-80^{\circ} \mathrm{C}$.

144 EV Isolation from AFSCs: The term AFSCs were grown to 70-80\% confluency, washed twice 145 with PBS and placed in serum free RPMI medium overnight. After $16 \mathrm{~h}$ of incubation, the 146 RPMI medium was recovered and centrifuged at $300 \mathrm{xg}$ for $5 \mathrm{~min}, 10,000 \mathrm{xg}$ for $30 \mathrm{~min}$,

147 followed by ultracentrifugation at $100,000 \mathrm{x}$ g for $2 \mathrm{~h}$ at $4^{\circ} \mathrm{C}$. The EV pellet was resuspended 148 in $1 \%$ DMSO/PBS and stored at $-80^{\circ} \mathrm{C}$.

149 Smart SEC purification: Size-exclusion chromatography was applied to samples destined for 150 miRNA arrays and ExoView analysis. Following differential ultracentrifugation, EV pellets were resuspended in $100 \mu \mathrm{l}$ of PBS and applied to Smart SEC columns (SSEC100A-1, BioNova, Madrid, Spain). The samples were then processed as recommended by the manufacturer's protocol.

154 Transmission electron microscopy: One drop of PBS (about $25 \mu 1$ ) containing EV at 5.8x $10^{8}$ 155 was placed on a 400-mesh holey film grid; after staining with $2 \%$ uranyl acetate (for 2 minutes)

156 the sample was observed with a Tecnai G2 transmission electron microscope (FEI Company, 
157 Hillsboro, OR, USA) operating at $100 \mathrm{kV}$. Images were captured with a Veleta digital camera

158 (Olympus Soft Imaging System, Tokyo, Japan).

159 NanoSight analysis: The size and concentration of EVs was determined by nanoparticle

160 tracing analysis (NTA) performed by NanoSight LM10 system (Malvern Panalytical, Malvern,

161 UK). Each vesicle sample was diluted (1:100) in PBS (filtered with a $0.22 \mu \mathrm{m}$ filter). For each

162 sample, a syringe pump flow of 30 was applied. Three videos of 60 seconds each were recorded

163 and analyzed, calculating an average number of EV size and concentration (particles/ml). All

164 samples were characterized with NTA 3.2 analytical software. The NTA settings were kept

165 constant between the samples.

166 Super-resolution microscopy: Super-resolution microscopy pictures of EVs were obtained

167 using a temperature-controlled Nanoimager S Mark II microscope from ONI (Oxford

168 Nanoimaging, Oxford, UK) equipped with a 100x, 1.4NA oil immersion objective, an XYZ

169 closed-loop piezo 736 stage, and $405 \mathrm{~nm} / 150 \mathrm{~mW}, 473 \mathrm{~nm} / 1 \mathrm{~W}, 560 \mathrm{~nm} / 1 \mathrm{~W}, 640 \mathrm{~nm} / 1 \mathrm{~W}$

170 lasers and dual or triple emission channels split at 640 / and $555 \mathrm{~nm}$. For sample preparation,

$171 \quad 10 \mu \mathrm{l}$ of $0.01 \%$ Poly-L-Lysine (Sigma-Aldrich, St. Louis, MO, USA) was placed on high-

172 precision coverslips previously cleaned in a sonication bath twice in $\mathrm{dH}_{2} \mathrm{O}$ and once in $\mathrm{KOH}$,

173 in silicon gasket (Grace Bio-Labs, Sigma-Aldrich). The coverslips were placed at $37^{\circ} \mathrm{C}$ in a

174 humidifying chamber for two hours. Excess of Poly-L-Lysine was removed. $1 \mu \mathrm{l}$ of EVs

$175\left(1 \times 10^{10}\right)$ resuspended in $9 \mu$ of blocking solution (PBS-5\% Bovine Serum Albumin) was

176 pipetted into a previously coated well to attach overnight at $+4^{\circ} \mathrm{C}$. The next day, the sample

177 was removed, and $10 \mu \mathrm{l}$ of blocking solution was added into the wells for $30 \mathrm{~min}$. Then, $2.5 \mu \mathrm{g}$

178 of purified mouse anti-CD9 conjugated with Atto 488 dye (ONI), anti-CD63 antibodies (SC-

179 5275, SC-31234, Santa Cruz, CA, USA) conjugated with Alexa Fluor 647 dye, HLA-G

180 antibody (SC-21799, Santa Cruz) conjugated with Alexa Fluor 555 dye, were prepared using 
the Apex Antibody Labelling Kit (Invitrogen) according to the manufacturer's protocol. One $\mu l$ of each antibody was added into the blocking buffer at a final dilution of 1:10. Samples were incubated in the dark at $+4^{\circ} \mathrm{C}$. The day after, samples were washed twice with PBS, and $10 \mu 1$ of the mixed ONI B-Cubed Imaging Buffer (Alfatest, Rome, Italy) was added for amplification of the EV signalling. Before each imaging session, bead slide calibration was performed for aligning the channels, to achieve a channel mapping precision smaller than $12 \mathrm{~nm}$. Images were taken in dSTORM mode using 30\% laser power for the $647 \mathrm{~nm}, 50 \%$ laser power for the 488 nm laser, and 30\% for the 555 channel. Two-channels (647 and 555) dSTORM data (5000 frames per channel) or three-channels (2000 frames per channel) $(647,555$ and 488) were acquired sequentially at $30 \mathrm{~Hz}$ (Hertz) in total reflection fluorescence (TIRF) mode. Singlemolecule data was filtered using NimOS software (v.1.18.3, ONI) based on the point spread function shape, photon count and localization precision to minimize background noise and remove low-precision and non-specific colocalization. Data has been processed with the Collaborative Discovery (CODI) online analysis platform www.alto.codi.bio from ONI and the drift correction pipeline version 0.2.3 was used. Clustering analysis was performed on localizations and BD clustering constrained parameters were defined (photon count 300-max, sigma 0-200 nm, p-value 0-1, localization precision 0-20 nm). Colocalization was defined by a minimum number of localizations for each fluorophore/protein within a distance of $100 \mathrm{~nm}$ or distance used from the centroid position of a cluster.

Western Blot: For protein analysis, EVs were lysed at $4{ }^{\circ} \mathrm{C}$ for $30 \mathrm{~min}$ in RIPA buffer $(20 \mathrm{nM}$

201 Tris- $\mathrm{HCl}, 150 \mathrm{nM} \mathrm{NaCl}, 1 \%$ deoxycholate, 0.1\% SDS, 1\% Triton X-100, pH 7.8) 202 supplemented with a cocktail of protease and phosphatase inhibitors and PMSF (Sigma203 Aldrich). For protein concentrations of less than $2 \mathrm{mg} / \mathrm{ml}$, a precipitation step was applied. The 204 pellet was resuspended in Laemmli Buffer and the sample was loaded onto the gel. As 205 determined by the Bradford method, aliquots of the cell lysates containing 10-15 $\mu \mathrm{g}$ of proteins 
were run on 4-20\% acrylamide gel SDS-PAGE under reducing conditions. The transfer of

207 proteins onto a PVDF membrane was performed using the iBlot ${ }^{\mathrm{TM}}$ Dry Blotting System (Life

208 Technology). Primary antibodies used were: CD81 at 1:200 (SC-31234), CD63 at 1:200 (SC-

209 5275), HLA-G at 1:200 (SC-21799), (all from Santa Cruz), CD105 at 1:1000 (362-820, Ancell)

210 and at 1:2000 calreticulin (2891S, Cell Signaling Technology, Danvers, MA, USA).

211 Chemiluminescent signal was detected using the ECL substrate (Bio-Rad), and bands were

212 detected by the ChemiDoc system (Bio-rad, Hercules, CA, USA).

213 MACSPlex: EV samples were tested with a bead-based multiplex analysis (MACSPlex

214 exosome kit, human, Miltenyi Biotec, Bergisch Galdbach, Germany). EV samples were diluted

215 with MACSPlex buffer to a final volume of $120 \mu \mathrm{l}$ with $5.8 \times 10^{8} \mathrm{EV}$ s per sample. Fifteen $\mu 1$ of

216 MACSPlex Exosome Capture Beads and $15 \mu 1$ of the antibody cocktail composed of APC

217 conjugated CD9, CD63 and CD81 were added to the buffer containing EVs and incubated on

218 an orbital shaker at $12 \mathrm{rpm}$ for 1 hour protected from light, at room temperature. After the

219 incubation, $1 \mathrm{ml}$ of MACSPlex buffer was added and the samples were centrifuged at 3,000 $\mathrm{g}$

220 for $5 \mathrm{~min}$. After a second wash, the samples were incubated for a further $15 \mathrm{~min}$ on the orbital

221 shaker. Thereafter the samples were centrifuged and prepared for flow cytometry analysis.

222 Median fluorescence intensity was calculated for all 39 bead populations. The analysis of the samples was performed by subtraction of the control and the fluorescence controls. For analysis of AFSC-EVs, the same method was followed with the substitution of the fluorescent antibody mix against tetraspanins (CD63, CD81 and CD9) with the APC labelled HLA-G antibody (130111852, Miltenyi Biotec, same volume of $15 \mu \mathrm{l}$ ). This method ensured that EVs attached to the $\mathrm{Ab}$ conjugated beads were only detected if positive for HLA-G.

228 ExoView array: For the analysis with the ExoView platform ${ }^{\mathrm{TM}}$ (NanoView Biosciences, 
collected fractions were applied directly to the ExoView chips. 10-35 $\mu$ l of the sample with a concentration of $5 \times 10^{8} \mathrm{EVs}$ was applied to a final volume of $35 \mu \mathrm{l}$ in the incubation solution.

232 EVs were then incubated overnight at room temperature. Immuno-fluorescence staining was

233 performed using antibodies provided by the company $(0.6 \mu 1$ of CD63, CD81, CD9). Alexa

234 Fluor 488 conjugated anti-CD117 (ab2164459, Abcam, Cambridge, UK), anti-Tie-2 (ab24859,

235 Abcam), anti-CD105 (362-820, Ancell) and HLA-G conjugated with Alexa Fluor 555 (SC-

21799, Santa Cruz) were used for detection of EV subpopulations. For all antibodies, the Apex

Antibody Labelling Kit was used (Invitrogen). All antibody conjugations were performed according to manufacturer's protocol. Chips were analyzed using the ExoView ${ }^{\mathrm{TM}} \mathrm{R} 100$ reader using ExoView Scanner software (v 3.0). The data were analyzed using ExoView Analyzer (v 3.0). Two sets of ExoView chips were tested. The first set of chips were pre-spotted with CD63/CD81/CD9, with addition of fluorescent antibodies CD63, CD81, CD9. The second set of chips were pre-spotted with CD63/CD81/CD9 with the addition of fluorescent antibodies HLA-G, CD117, Tie-2 and CD105.

Angiogenesis: Human umbilical vascular endothelial cells (HUVEC) were bought from ATCC (ATCC-PCS-100-010, Manassas, VA, USA). Cells were cultured until the 6th passage in complete EBM medium (Lonza, Basel, Switzerland). Cell medium was changed three times per week, and cells were passaged when they reached 70-80\% confluency. The cells were split by washing in PBS, then applying $2 \mathrm{ml}$ of $10 \%$ trypsin-EDTA and re-plated on T75 flasks coated with attachment factor (S-006-100, Gibco). In vitro formation of capillary-like 250 structures was performed on growth factor-reduced Matrigel (356231, Corning, NY, USA). 251 HUVEC cells were mixed with EVs (1000 EVs/cell) and/or anti-CD105 Ab TRC-105 $8 \mu \mathrm{g} / \mathrm{ml}$ 252 (TRACON Pharmaceuticals, CA, USA) and recombinant soluble CD105 (s-Endoglin, 100 ng/ml; C-60059, PromoKine, Heidelberg, Germany) in complete EBM medium and seeded at a density of $15 \times 10^{3}$ cells per well on a 24 -well plate. For combination of EVs with the anti- 
CD105 Ab TRC-105, both components were mixed and incubated for $25 \mathrm{~min}$ at room temperature, prior to adding them to the cell suspension. Cells were periodically observed with a Nikon TE2000E inverted microscope (Nikon, Tokyo, Japan), and experimental results were recorded after $16 \mathrm{~h} ; 5$ images were taken per well. Image analysis was performed with the ImageJ software v.1.53c, using the Angiogenesis Analyzer. The data from three independent experiments were expressed as the mean \pm SD of tube length in arbitrary units per field.

miRNA array analysis: Total EV RNA was extracted from 3 normal and 3 preeclamptic amniotic fluid samples after ultracentrifugation and SEC. All samples were processed

following the manufacturer's protocol. The RNA concentration was measured using NanoDrop (NanoDrop ND-1000, ThermoFisher) and precipitated to a final concentration of $10 \mathrm{ng} / \mu \mathrm{l}$. The total cDNA was pre-amplified prior to miRNA quantification. Expression of 754 different miRNAs was evaluated by quantitative real-time PCR using TaqMan Array MicroRNA card A (v.3.0) and B (v.3.0) and according to Megaplex manufacturers protocol. All the samples were run separately. Quantification was performed using a QuantStudio 12K Flex Real-Time PCR System (Applied Biosystems, Waltham, MA, USA). Ct values were analyzed by Expression Suite software v1.3 using global normalization. A maximum threshold of 35 was applied for analysis of EVs from normal pregnancies in comparison to preeclampsia.

273 Quantitative real-time PCR: Validation of the miRNAs was performed by quantitative real-

274 time PCR (qRT-PCR) on 3 different amniotic fluid EV samples for normal pregnancy and 275 preeclampsia. The miRNAs were reverse-transcribed into cDNA using a High Capacity cDNA 276 reverse transcription kit (Applied Biosystems) following the manufacturer's protocol. The 277 qRT-PCR was performed using 20 miRNA primers (Supplementary Table 1) and miScript SYBR Green PCR Kit (Qiagen, Hilden, Germany). RNA U6 was used as the endogenous 
control. The samples were analysed using the comparative CT method. In total 20 miRNAs

were selected and validated, based on literature research via Pubmed interrogation with the

following keywords: "miRNAx" and "angiogenesis or placenta or pre-eclampsia" or

"miRNAx" and "angiogenesis or placenta or pre-eclampsia" and extracellular vesicles".

Acquisition of PE-related miRNAs gene targets and transcription factors and network

visualization: We obtained $\mathrm{PE}$ associated miRNAs based on previously mentioned

experiments. miRTarBase v8.0 was used to identify gene targets and extract miRNA-gene pairs

(Huang et al., 2020). To minimize false positives, only strong-evidence miRNA-target pairs

were considered. Literature-curated transcription factor (TF)-miRNA pairs of deregulated

miRNAs were extracted from TransmiR (Tong et al., 2019) miRNA, target gene, and TF

interaction pairs were visualized in Cytoscape v3.8.2 (Shannon et al., 2003). The interactions

between genes and TF, and interacting genes were integrated using the Cytoscape App

Bisogenet v3.0.0 (Martin et al., 2010).

Network enrichment analysis: The analysis of overrepresented gene ontology (GO)

biological process terms and pathways associated with the target genes, was performed using

EnrichR webtools (Chen et al., 2013). Ontology and pathway GO terms with an adjusted p-

value $<0.05$ were considered significantly overrepresented. In addition, the Cytoscape App

BinGo was used to visualize and cluster the gene annotation terms into groups (Maere,

297 Heymans and Kuiper, 2005). BinGo results were corrected for multiple testing using

298 Benjamini-Hochberg false discovery rate correction. The KEGG, Reactome and WikiPathways

299 databases, as well as the Tissue Expression ArchS4 databases, were used to obtain specific

300 gene annotations (Ogata et al., 1999; Rouillard et al., 2016; Jassal et al., 2020). The comparison

301 of miRNA-gene target interactions and GO biological processes to previous studies (Pillay et 
al., 2009). GO biological process function terms with an adjusted $\mathrm{p}$ value $<0.001$ after

304 Bonferroni step down correction and term fusion were considered.

305 Synergistically working miRNAs: MiRNAs with binding sites near each other (between 13-

30635 nucleotides) can cooperate in target repression. We identified cooperating miRNA pairs of

307 upregulated preeclampsia-associated miRNAs using the TriplexRNA database

308 (https://triplexrna.org/). The results were filtered for targets involved in angiogenesis based on

309 GO terms (Lai et al., 2012).

310 Statistical tests: All statistical analyses were performed using GraphPad Prism software (v.

311 8.00; GraphPad, CA, USA). For comparisons of each group, an unpaired Student's t-test was

312 used. FWelch's correction was performed when the variance across groups was assumed to be

313 unequal. Differences were considered significant when the p-value was $<0.05$. For multiple

314 comparison analyses, ANOVA with Bonferroni multicomparison post hoc test. A two-tailed $p$ -

315 value $<0.05$ was considered statistically significant. 


\section{Results}

\section{Characterization of amniotic fluid-derived EVs}

318 EVs were isolated from term amniotic fluid of 40 normal pregnancies (Table 1) by differential

319 ultracentrifugation followed by filtration and, for selected experiments (miRNA and ExoView

320 analysis), by size-exclusion chromatography. The normal pregnancy EVs (NP-EVs) were first

321 characterized based on their size and concentration using nanoparticle tracking analysis (Figure

322 1A). Homogeneous concentration and particle size were obtained among the different samples,

323 with a mean size of $221.8 \pm 6.2 \mathrm{~nm}$. Western Blot analysis confirmed the expression of EV

324 specific markers (CD63, CD81) and revealed the presence of the placental maker HLA-G,

325 previously shown to characterize placental-derived EVs (Orozco et al., 2009) (Figure 1B). The

326 absence of the cytoplasmic marker calreticulin showed a lack of cellular contamination (Figure

327 1B). TEM showed heterogeneous EV populations (particles ranging from approximately 50 to

328 approximately $200 \mathrm{~nm}$ in diameter) (Figure 1C). To quantify the expression of CD63 and HLA-

$329 \mathrm{G}$ and to assess their possible colocalization, we performed super-resolution imaging of NP-

330 EVs using dSTORM. More than 6,000 single-EV images were acquired and analyzed.

331 Clustering analysis showed that the majority (around $87 \%$ ) of $\mathrm{CD}^{2} 3^{+}$EVs also expressed HLA-

332 G (Figure 1D). 

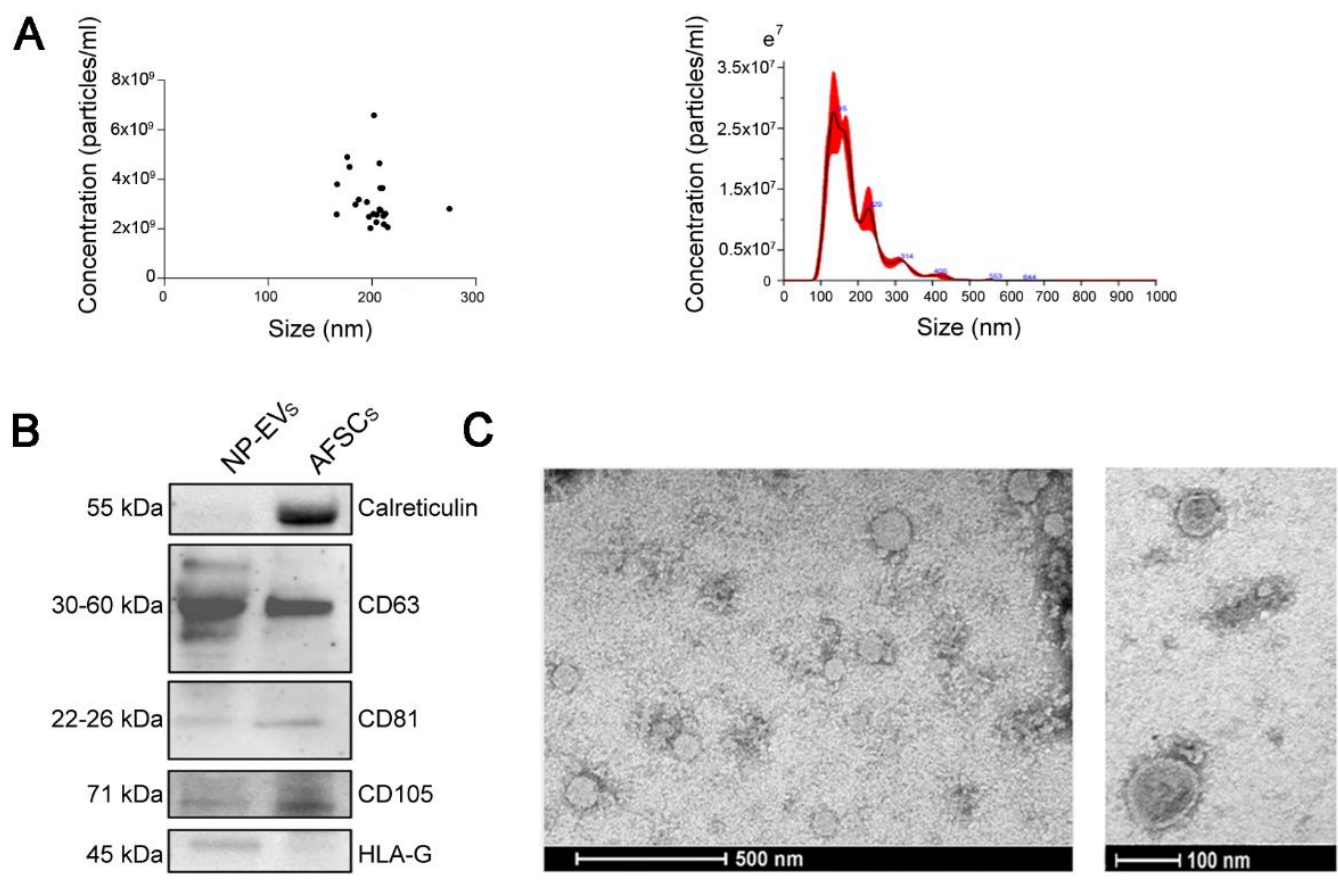

D
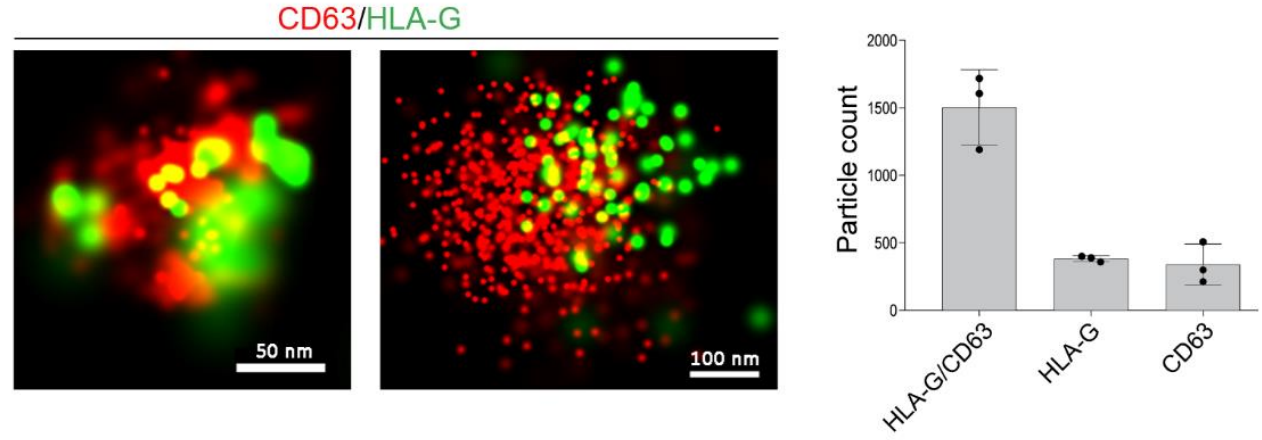

334

335

336

337

338

339

340

341

342

343

344

345

346

347

348

349

350

Figure 1. Characterization of NP-EVs. A) Nanoparticle tracking analysis of NP-EVs $(n=30)$ showing homogenous size and concentration. Dilution of 1:100 in PBS in $1 \mathrm{ml}$ was used to test the samples (left panel). A representative graph showing size distribution of a NP-EV sample (right panel). B) Representative Western Blot image showing the presence of CD63, CD81, CD105 and HLA-G in NPEVs (10-15 $\mu \mathrm{g}$ proteins) and in AFSC lysate (10-15 $\mu \mathrm{g}$ proteins) and the presence of calreticulin in cell lysate only. Three different NP-EV samples were tested with similar results. C) Representative transmission electron microscopy images at low- and high-power fields of NP-EVs showing heterogenous EV population. D) Representative super-resolution microscopy images of single amniotic fluid-derived EVs expressing CD63 (red) and HLA-G (green). The number of single and double positive EVs for CD63 and HLA-G was analysed in 3 NP-EV preparations using the CODI software; the graph shows the mean $\pm \mathrm{SD}$ of a cumulative analysis of 10 fields for each preparation, total EV number: 6,676 .

In addition, super-resolution microscopy was used to analyze the size and expression of tetraspanin markers (CD63, CD81 and CD9) at a single-molecule level in NP-EVs (Figure 2).

The results showed that NP-EVs were a heterogeneous population with highly variable tetraspanin expression (Figure 2E). The analysis of more than 20,000 EVs using a dedicated 
352 software (https://alto.codi.bio/) showed that the majority of EVs were either triple positive for

353 CD63, CD81 and CD9 or double positive for CD81 and CD9, with lower fractions expressing

354 double or single tetraspanins (Figure 2E). The overall average particle size evaluated by the

355 super-resolution microscope was $121 \pm 26 \mathrm{~nm}$. This measurement, based on specific EV

356 detection using tetraspanins, overlaps the results obtained by transmission electron microscopy.

357 The larger EV size detected by nanoparticle tracking analysis can be possibly explained by the

358 evaluation of the EV hydrodynamic size, under solution using this technique, in comparison

359 with the measurement of the dry radius with microscopy techniques (Montis et al., 2017). In

360 addition, possible artifacts including EV aggregates or non-EV particles can be detected by

361 nanoparticle tracking analysis, suggesting that it should be applied for size evaluation together

362 with other side techniques, as previously described (Skovronova et al., 2021). 
A

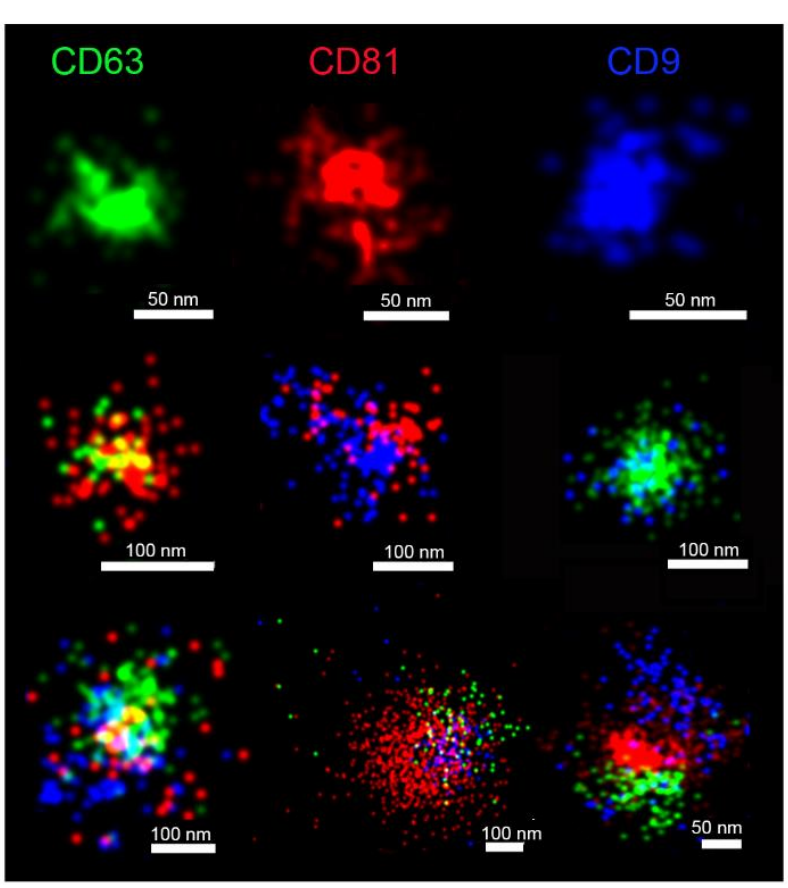

B

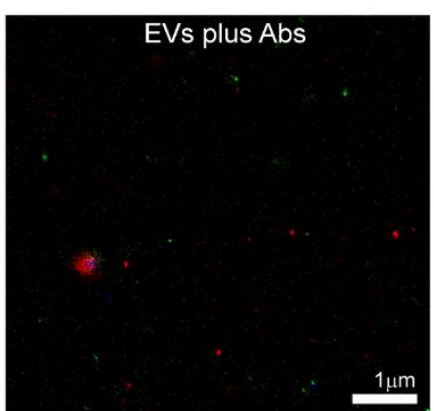

C

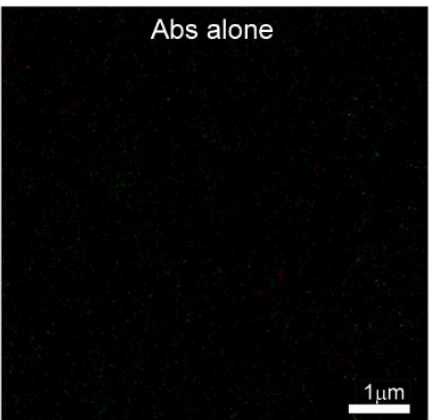

D
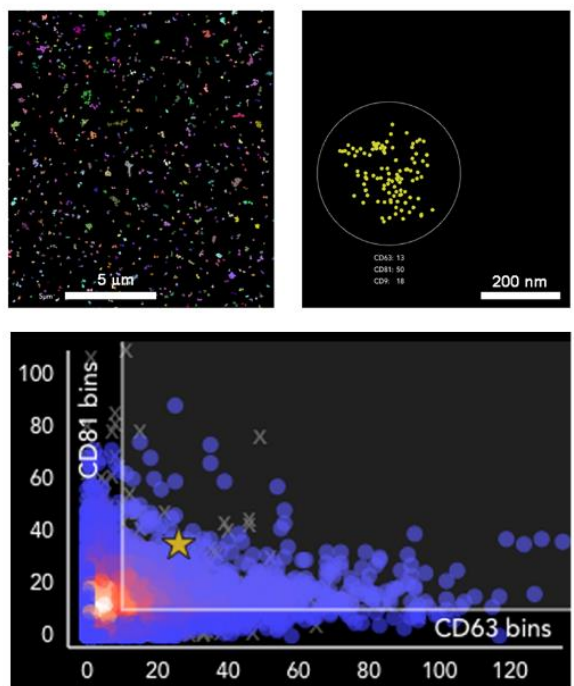

$E$

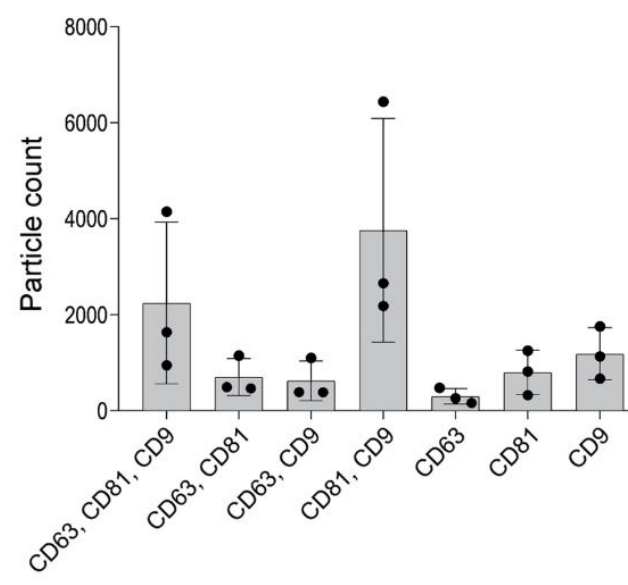

Total particle count: 22,487

Average particle size: $121 \pm 26 \mathrm{~nm}$

Figure 2. Super-resolution microscopy analysis of tetraspanins expression in NP-EVs.

A) Representative super-resolution microscopy images of NP-EVs showing single, double, and triple expression of CD63 (green), CD81 (red), CD9 (blue). The corresponding scale bare is below each EV image. B) Representative super-resolution images, at low magnification, showing staining with antitetraspanin antibodies in the presence of EVs (EVs plus Abs) or C) in the absence of EVs (Abs alone). D) Representative clustering strategy of NP-EV analysis showing a large field of view (left panel), a selected cluster (right panel) and a graph (bottom panel) of CD81/CD63 cluster distribution. E) Clustering analysis of super-resolution microscopy images showing the single, double, and triple positive EV fractions expressing the tetraspanin markers. The analyses were performed in $3 N P-E V$ preparations using a CODI software; the graph shows the mean $\pm \mathrm{SD}$ of a cumulative analysis of 10 


\section{Comparison of amniotic fluid EVs from normal and preeclamptic pregnancies}

378 EVs were also isolated from term-amniotic fluid of 7 preeclamptic pregnancies (Table 1). The

379 different volumes and EV concentrations in each sample are reported in Supplementary Table

380 1. No difference in mean EV concentration between NP-EVs and PE-EVs $\left(2.85 \times 10^{9}\right.$ and

$2.17 \times 10^{9}$ particles $/ \mathrm{ml}$, respectively) was observed, although the relevance of this parameter is

382 limited by the dynamic nature of this biofluid (Underwood, Gilbert and Sherman, 2005). As amniotic fluid contains EVs originating from several sources, a comprehensive analysis of EV surface receptors characterizing several cell types was performed using MACSPlex, a beadbased cytofluorimetric kit able to perform a semiquantitative fluorescent analysis of 39 different EV surface markers. Figure 3A shows the most relevant markers expressed. EVs within the amniotic fluid of both normal and preeclamptic pregnancies highly expressed tetraspanins, as confirmed with the methods shown above. In addition, the typical amniotic fluid marker, CD24 (Keller et al., 2007) and CD133, known to be highly expressed by urine EVs (Dimuccio et al., 2014) were present at a high level in both EV types, indicating the presence of EVs derived from fetal urine. Expression of the embryonic stem and epithelial cell marker CD326 was detected in both samples. Interestingly, a significantly higher expression

393 of the progenitor/mesenchymal marker SSEA-4 and CD105 was detected in PE-EVs (Figure 3A). No expression of leukocyte (CD3, CD4, CD8, CD45, CD56, CD19), endothelial (CD31) or platelet (CD42a and CD49e) markers was observed (data not shown).

396 ExoView analysis was subsequently used to assess at single-EV level the expression of NP-

397 EV and PE-EV surface markers of interest. After EV affinity binding to tetraspanins, CD105, 398 Tie-2, CD117 (c-kit) and HLA-G expression were evaluated on captured EVs (Figure 3C). The 399 analysis confirmed the presence of HLA-G, as shown by super-resolution microscopy, and 400 revealed the presence of the angiogenic marker Tie- 2 and of the stem cell marker CD117 on 
403 with the other makers, we were able to assess their co-expression. The analysis showed that

404 the HLA-G expressing EVs displayed similar CD105 and c-kit markers levels, suggesting that 405 the increased CD105 expressing EVs present in PE were not of placental origin (Figure 3D).

406 At variance, Tie-2 levels were significantly lower in placental EVs of PE pregnancies. No 407 differences in tetraspanin levels, used as control, were observed (Supplementary Figure 1B).

408 The increased CD105 expression in PE-EVs with respect to NP-EVs was also confirmed by 409 Western Blot analysis (Supplementary Figure 2). 
A

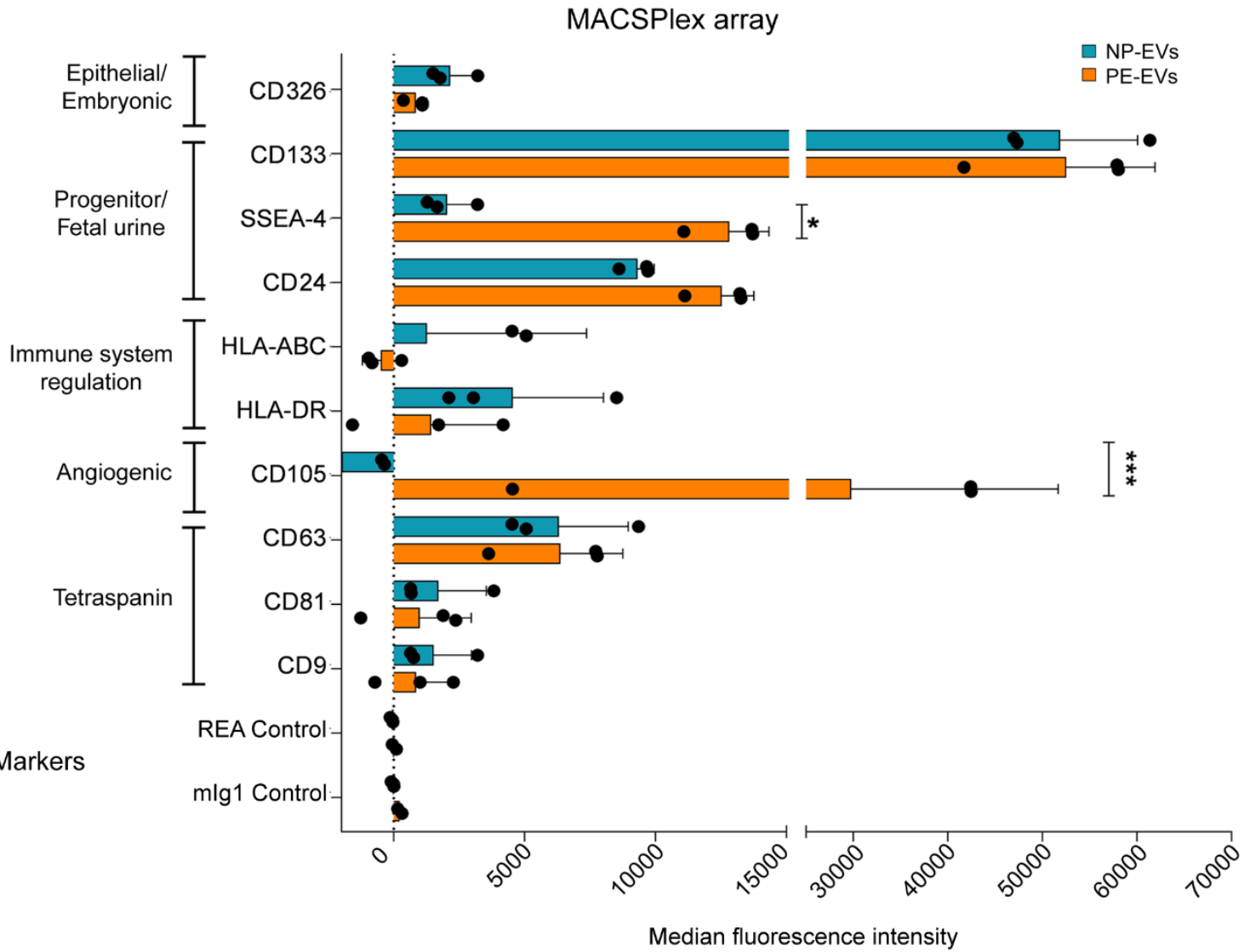

B

C
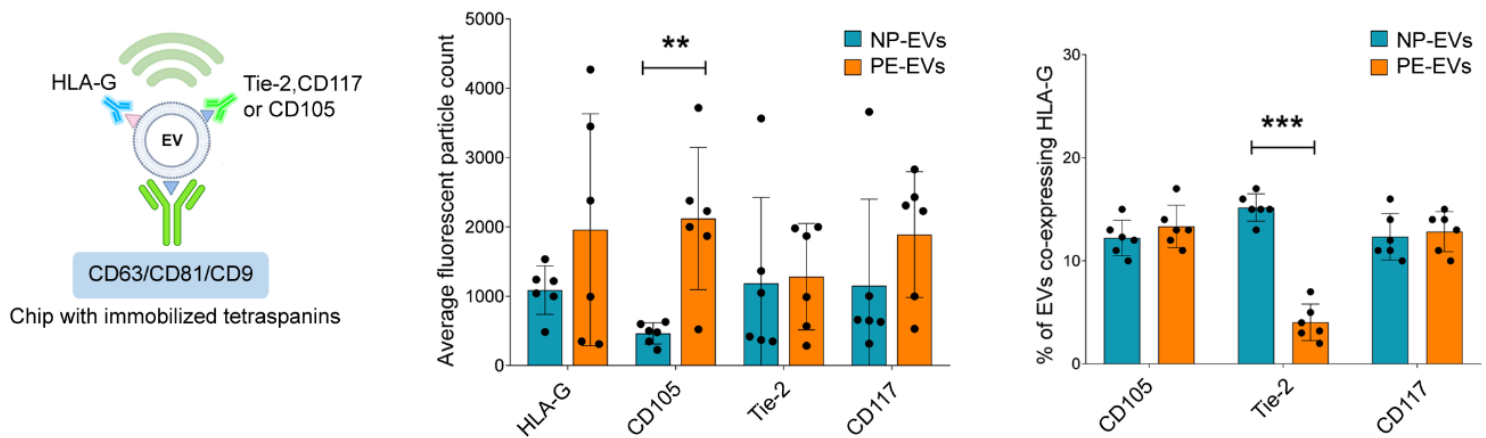

411 Figure 3. Increased CD105 expression in PE-EVS. A) MACSPlex analysis showing the median 412 fluorescence intensity of surface markers characteristic of EVs (tetraspanins) or different cell of origin, 413 expressed by NP- and PE-EVs (n=3). Expression of CD105 and SSEA-4 was significantly increased in

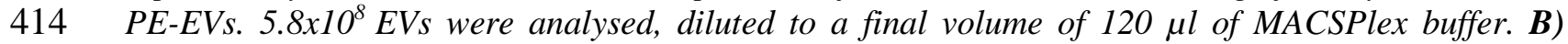
415 Diagram explaining the experimental method behind ExoView technology in relation to the graphs in 416 panel C. C and D) ExoView analysis of amniotic fluid-derived NP-EVs $(n=6)$ and PE-EVs $(n=6)$. C) 417 Comparison of the expression of HLA-G, Tie-2, CD105 and CD117 (c-kit) shown as average fluorescent 418 particle count in NP-EVs vs PE-EVs from combined tetraspanins capture of CD63, CD81 and CD9. D) 419 Normalized expression of HLA-G positive EVs co-expressing other angiogenic (CD105 and Tie-2) and 420 stem cell (CD117) markers. $5.8 \times 10^{8}$ EVs in final volume of $35 \mu l$ of buffer were used for all samples. Unpaired Student's $t$-test $*=P<0.05, * *=P<0.01, * * *=P<0.001$ 
422 Finally, we tested the characteristics of EVs derived from cultured term AFSCs, as a possible

423 source of amniotic fluid-EVs (Figure 4). Detection of AFSC-derived EVs captured on

424 tetraspanin coated chips revealed the presence of distinct subpopulations of HLA-G and CD105

425 expressing EVs, being $\mathrm{CD} 105^{+}$EVs the larger fraction. No co-expression of the two markers

426 was observed (Figure 4A and B). Analysis of the HLA-G ${ }^{+}$AFSC-EV fraction by MACSPlex

427 analysis confirmed the lack of CD105 expression (Figure 4C and D). These data suggest that

428 the $\mathrm{CD} 105^{+}$EV fraction observed in PE-EVs could possibly derive from cells of fetal or fetal membrane origin, including AFSCs. 
A

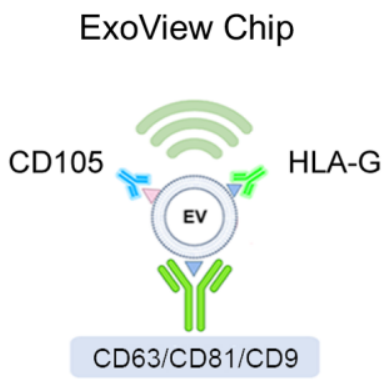

C

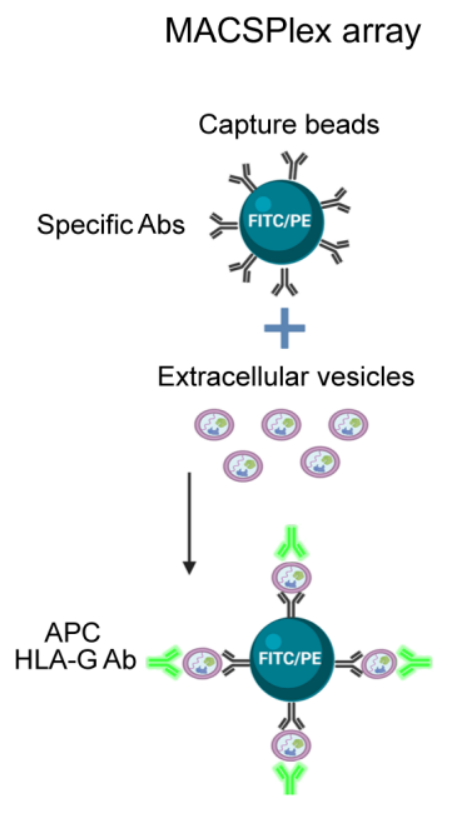

B

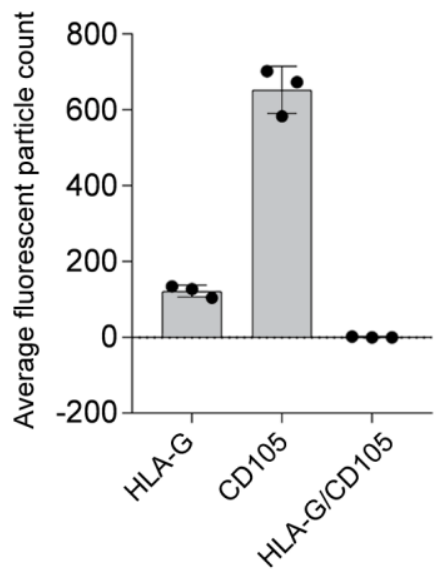

D

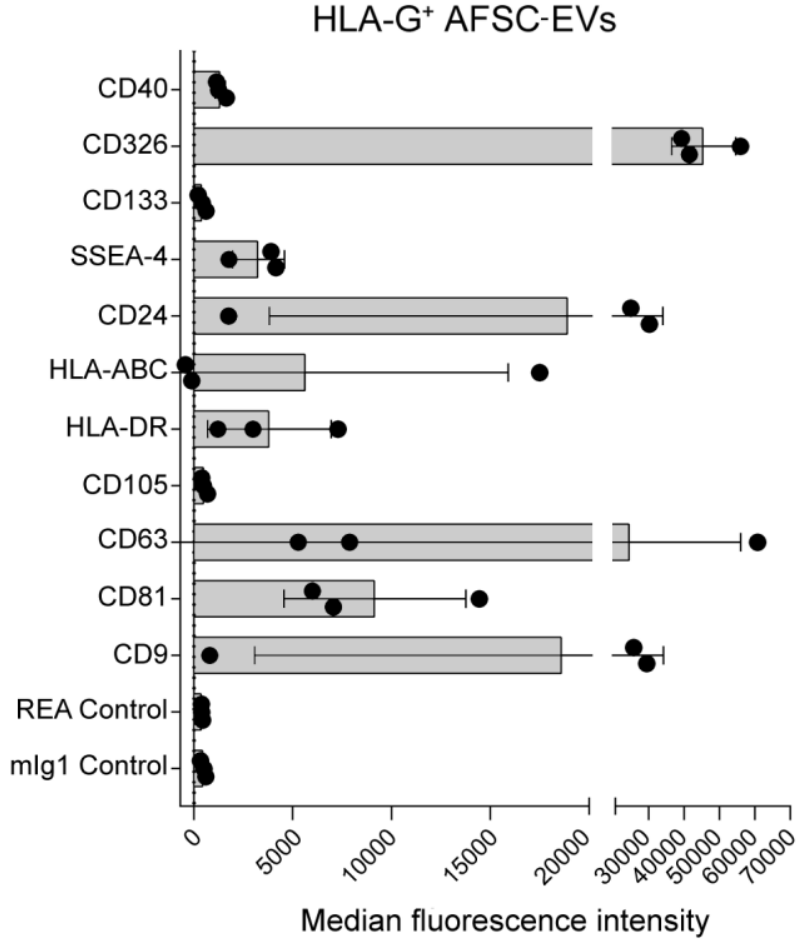

Figure 4. Characterization of HLA-G-expressing EVs from term AFSCs. A) Diagram explaining the experimental method in relation to the graph in panel B. B) Fluorescent particle count of AFSC-EVs captured on tetraspanin-coated chip analyzed by ExoView, showing expression of $\mathrm{HLA}^{-G^{+}}$and $\mathrm{CD} 105^{+}$ $E V s$, but lack of co-expression of the markers. C) Graphical representation of MASCPlex analyses to characterize HLA-G ${ }^{+}$AFSC-EVs, using Ab-coated fluorescent beads and APC-labelled anti-HLA-G $A b$. D) The graph shows the median fluorescence intensity of surface markers co-expressed on HLA$G^{+}$AFSC-EVS ( $\left.n=3\right)$. AFSC-EVs $\left(5.8 \times 10^{8}\right)$ were gated for the HLA-G positivity and analysed for the $C$ were created with https://biorender.com. 


\section{Functional characterization of NP-EVs and PE-EVs on angiogenesis}

442 Soluble endoglin is a differentially spliced form of endoglin and is a relevant antiangiogenic

443 factor in PE, acting as a decoy receptor for transforming growth factor beta (TGF- $\beta$ ) (Valluru

444 et al., 2011). To test the angiogenic properties of NP-EVs, PE-EVs, and the possible role of

445 CD105 increased expression on the EV surface, a tube formation assay was performed.

446 Amniotic fluid-derived EVs both from NP and PE, showed a significant reduction in the

447 organization of the HUVEC into capillary-like structures on Matrigel, with respect to the

448 positive control, with the PE-EVs being significantly more effective than NP-EVs in reducing

449 tube formation. To test the relative role of the increased expression level of CD105 on the

450 surface of PE-EVs, TRC-105, an anti-CD105 monoclonal Ab used as an antiangiogenic drug

451 (Brossa, Buono and Bussolati, 2018), was applied. We found that the addition of TRC-105 to

452 PE-EVs, but not to NP-EVs, prior to HUVEC stimulation significantly abolished their inhibitory effect (Figure 5), indicating the role of surface CD105 in angiogenesis reduction. 
A

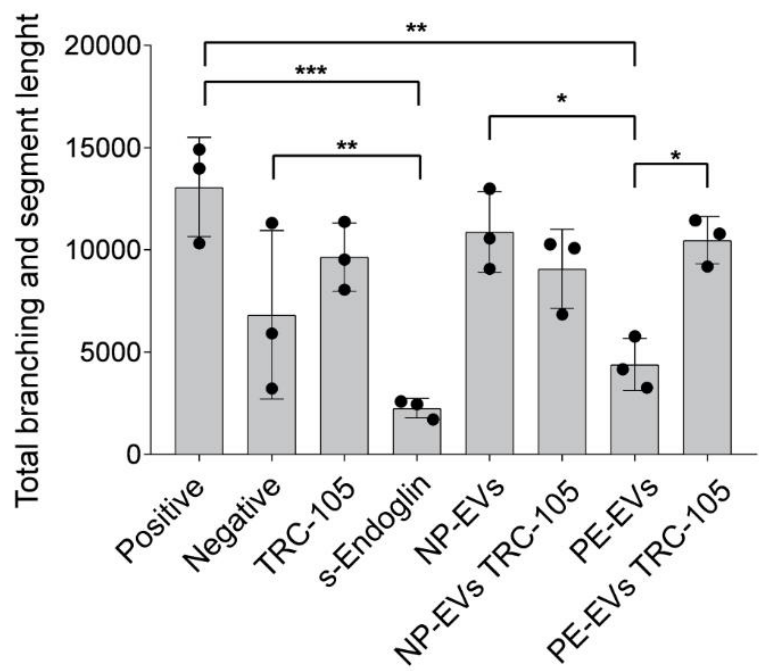

B
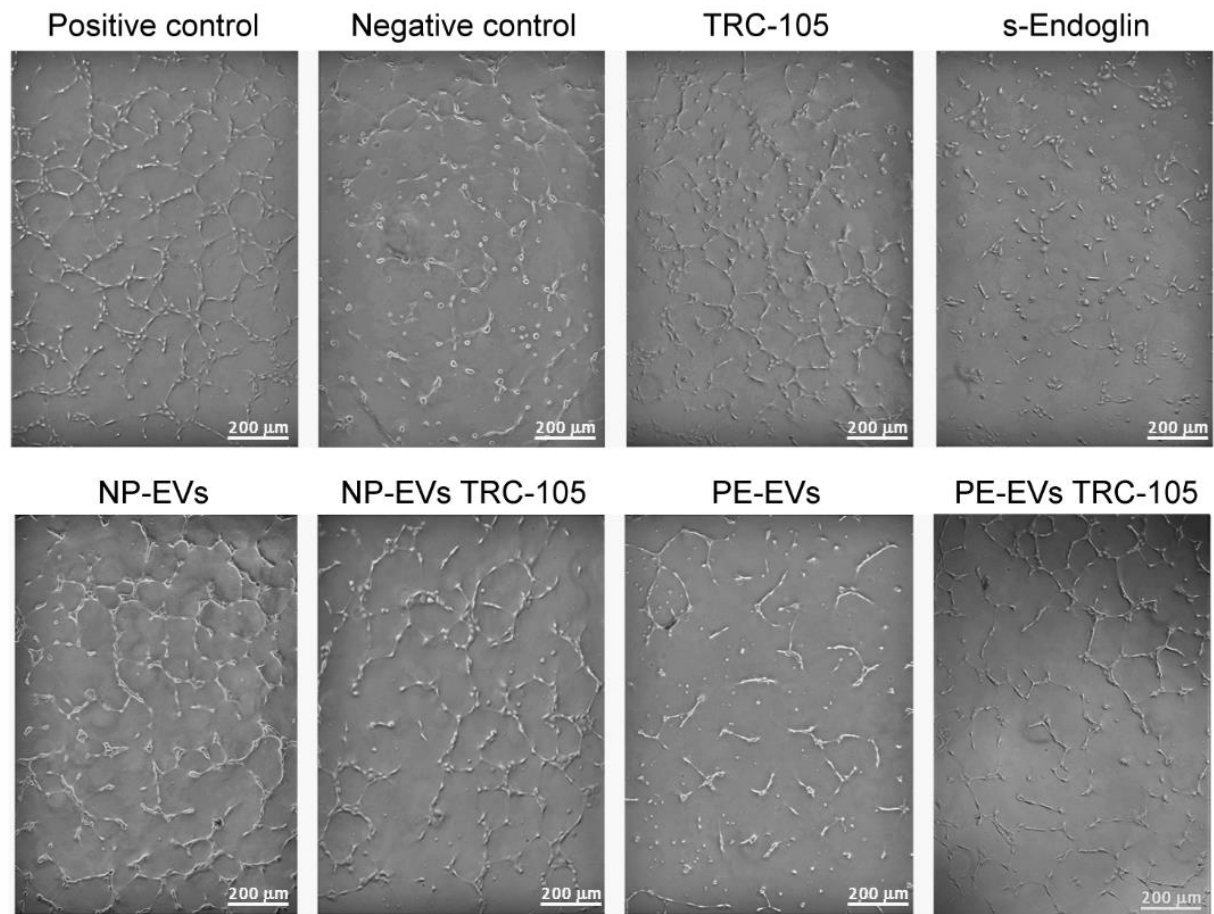

PE-EVs TRC-105

455

Figure 5. Effect of NP-EVs and PE-EVs on tube formation and role of CD105. A) Quantification of tube lengths of HUVEC treated with NP-EVs, PE-EVs with or without anti-CD105 Ab (TRC-105) (8 $\mu \mathrm{g} / \mathrm{ml})$. A concentration of $1000 \mathrm{EVs} / \mathrm{cell}$ was used. Positive control: complete EBM medium; negative control: EBM medium without FBS. s-Endoglin (100 $\mathrm{ng} / \mathrm{ml})$ was used as control for angiogenesis inhibition. Statistical analysis was performed using ANOVA with Bonferroni's post-hoc test. $*=P<0.05$, $* *=P<0.01, * * *=P<0.001$. B) Representative images of the tube formation assay. 


\section{Differential miRNA expression in NP-EVs and PE-EVs}

466 In order to identify the key regulatory miRNA in amniotic-derived EVs and their possible

467 differential expression in PE-EVs, we performed miRNA arrays on SEC-purified EV

468 preparations from $3 \mathrm{NP}$ and $3 \mathrm{PE}$ amniotic fluids. A large number of miRNAs were present in

469 amniotic fluid-EVs. The 3 sample repeats were combined for ease of interpretation. The raw

470 data can be found at https://fairdomhub.org/projects/244, showing miRNA expression for each

471 individual sample. The top and bottom 10 expressed miRNAs in NP-EVs are shown in

472 Supplementary Table 2. Overall, 46 miRNAs were detected as differentially expressed in PE-

473 EVs vs NP-EVs (Figure 6A and B). The complete list of miRNAs can be found at

474 https://fairdomhub.org/data_files/4182?version=1. In particular, 20 miRNAs of interest were

475 selected for qRT-PCR validation as previously described in EVs, biological fluids or placental

476 tissue from preeclamptic women from literature analysis (Supplementary Table 4). Results

477 confirmed a deregulated trend of the majority of miRNAs in PE-EVs, with let-7e, miR-15b,

478 miR-195,miR-613, miR-26a and miR-144 showing significance (Figure 6C). 
bioRxiv preprint doi: https://doi.org/10.1101/2022.02.14.480331; this version posted February 14, 2022. The copyright holder for this preprint (which was not certified by peer review) is the author/funder, who has granted bioRxiv a license to display the preprint in perpetuity. It is made available under aCC-BY-NC-ND 4.0 International license.

A

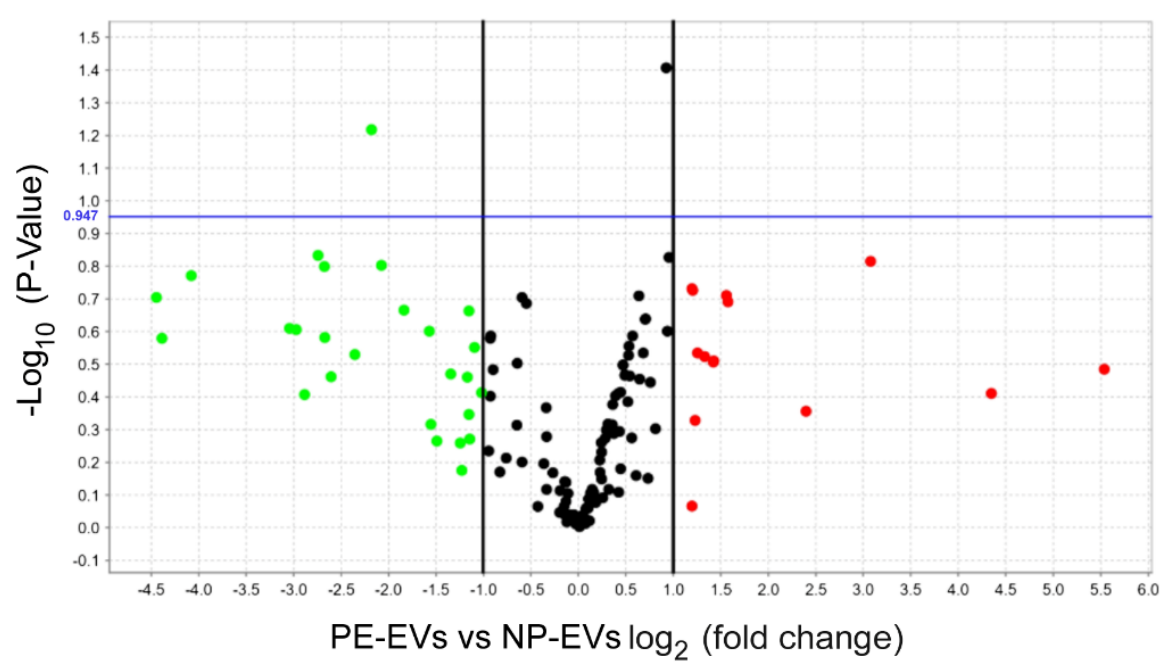

B

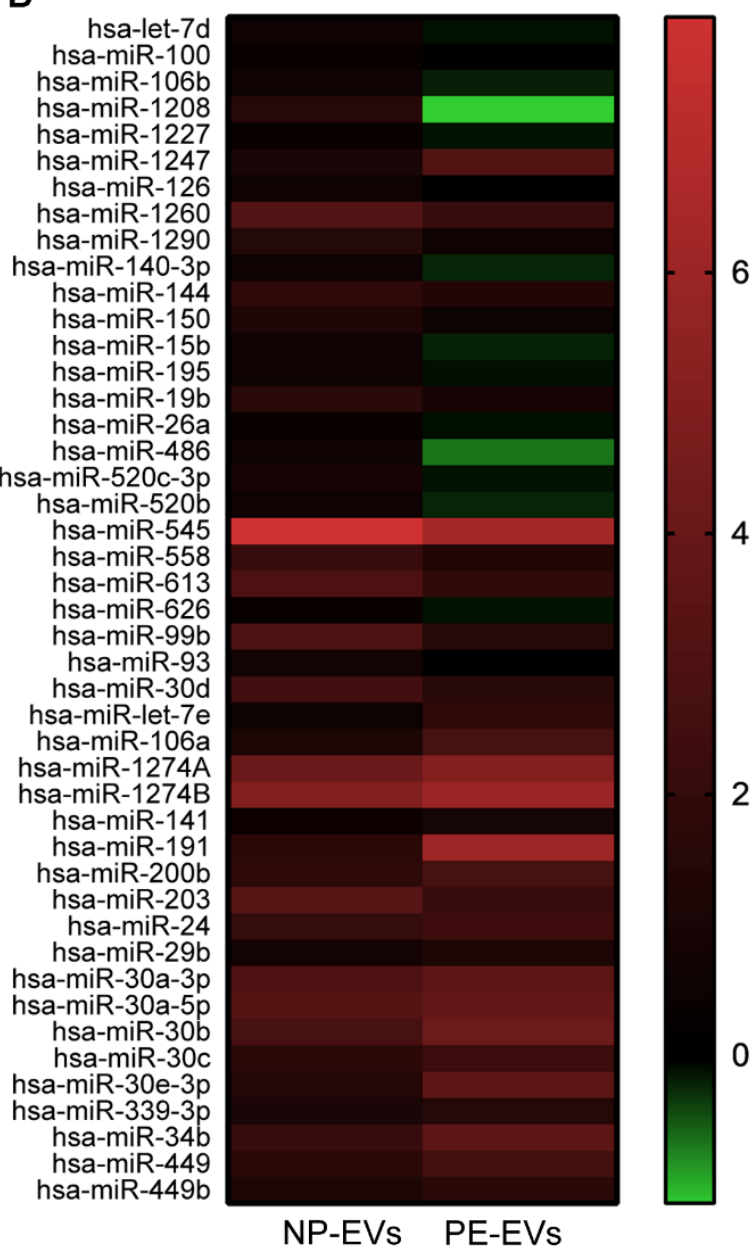

C

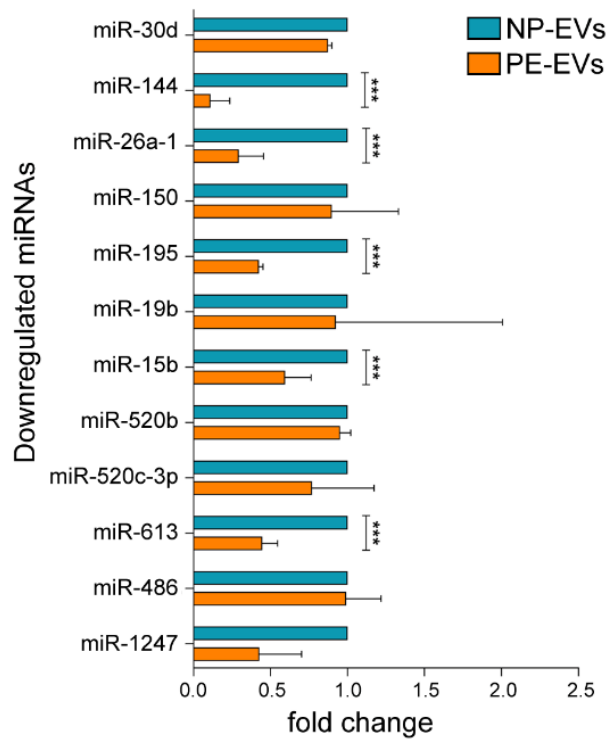

2

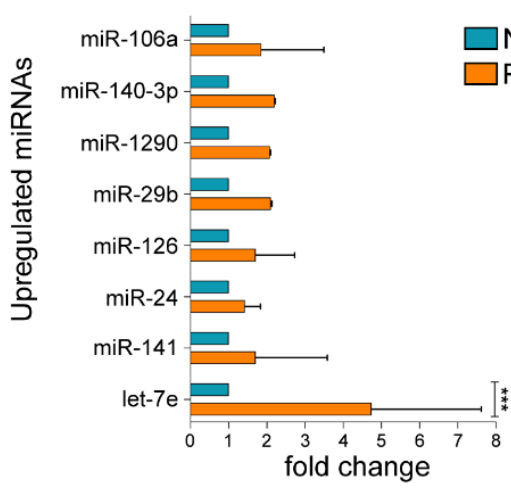

Figure 6. MiRNA analysis of NP-EVs and PE-EVs. A) Volcano plot showing the differential expression of miRNAs derived from NP-EVs vs PE-EVs. B) Heatmap showing the miRNAs with the highest fold changes between the samples. C) Validation of the deregulated miRNAs, normalized to RNU6 and CTL, as evaluated by qRT-PCR analysis. Data are expressed as mean $\pm S D$ of at least three different experiments normalized to CTL and to one, with let-7e, miR-195, miR-613, miR-15b, miR-26a1 , and miR-144, showing significant regulation differences. Unpaired Student's $t$-test: $* * *=P<0.001$. 


\section{Bioinformatic analysis}

487 The search of deregulated miRNA gene targets revealed more than 1000 strong evidence miRNA-gene interactions, involving 667 unique genes, with the vascular endothelial growth

489 factor (VEGF) gene being central in the interactome (Supplementary Figure 3). Further

490 functional enrichment analysis of GO terms and pathways supported the overall miRNA

491 involvement in the regulation of angiogenesis and vascular remodeling (Supplementary Figure

4924 and 6).

493 Accordingly, the analysis of target tissue and cell specific gene expression profile identified,

494 among the top represented, myoblast, omentum and placenta (Supplementary Figure 5).

495 Finally, analysing for miRNAs acting on a common target and resulting in an increased

496 inhibitory effect via triplex analysis, COL22A1, coding for collagen XXII, appeared to be

497 targeted by cooperative miRNAs with the lowest predicted repression (Supplementary Figure

498 6). Further details can be found in the bioinformatic section of supplementary data. The

499 complete list of miRNA gene targets and network enrichment results from EnrichR and BinGo

500 can be found at: https://fairdomhub.org/projects/244.

501 


\section{Discussion}

504 As reproductive EV research advances, investigation of placenta, surrounding gestational tissues, and amniotic fluid is of profound importance, in order to understand the factors involved in fetal development and maternal health. This paper provides for the first time a deep

507 characterization of EVs present in term amniotic fluid in normal and preeclamptic pregnancies.

508 Using several orthogonal techniques (chip-based platform, cytofluorimetric bead-based multiplex assay and super-resolution microscopy analysis), we show that EVs of term amniotic fluid represent a heterogeneous population of EVs of multiple origins, including placental

511 tissues, fetal urine, and stem cells. Moreover, we identified potential antiangiogenic properties

512 of amniotic fluid-EVs from preeclamptic pregnancies, supported by the specific upregulation 513 of the CD105 surface marker and the deregulation of several angiogenic miRNAs (Figure 7).

514 We here characterized EVs isolated from term amniotic fluid using sub-sequential 515 centrifugation, and we further purified them for the identification of specific surface receptors and miRNA cargo using size-exclusion chromatography to remove contaminating factors

517 possibly present in the amniotic fluid (Monguió-Tortajada et al., 2019; Théry et al., 2018). The

518 characterization of NP-EVs at a single-EV level revealed a highly heterogeneous population of

519 EVs with variable tetraspanins expression. These results are in agreement with those reported 520 by Han and colleagues on colocalization analysis of tetraspanins in EVs derived from different cell lines, showing distinct fractions of single, double or triple co-expressing EVs (Han et al., 2021).

Moreover, based on surface marker expression as well as miRNA content, we found that EVs

524 within the term amniotic fluid may originate from various sources. Indeed, amniotic fluid-EVs expressing CD24 were mainly derived from fetal urine (Keller et al., 2007). In this study, we confirmed the possible cellular origin of the EVs attributed to fetal urine by expression not only 
of CD24 but also of CD133, which is also considered to be highly present in urinary EVs

528 (Dimuccio et al., 2014). Additionally, amniotic fluid-EVs expressed mesenchymal and stem markers, possibly suggesting the origin from $\mathrm{CD} 117^{+}$amniotic fluid stem cells (Cananzi, Atala and De Coppi, 2009), amniotic CD105' mesenchymal stem cells (Baghaei et al., 2017), and SSEA-4 ${ }^{+}$expressing embryonic stem cells (Noisa et al., 2012). Interestingly, we also showed that a large fraction of amniotic NP-EVs expressed HLA-G, as detected by ExoView and superresolution microscopy. HLA-G, the distinct HLA isoform involved in maternal tolerance of the fetus, was previously reported to characterize EVs from the placenta in vitro or in vivo in the maternal circulation (Eunsung Mouradian, 2008; Orozco et al., 2009). It is also conceivable that the reported presence of HLA-G within the amniotic fluid could be related to its expression on the EV membrane (McMaster et al., 1998). The origin of the HLA-G expressing EVs in the amniotic fluid is unclear. They could possibly directly originate from the amniotic membrane surrounding the fetus and containing the amniotic fluid. Another possible explanation is the passage of trophoblast-derived EVs through the chorion and amnion to reach the amniotic fluid

541 (McMaster et al., 1998). The placental origin of amniotic fluid-EVs is also suggested by the analyses of the deregulated miRNA target genes in preeclampsia, showing the most represented cell targets origin from the myoblast, the omentum and the placenta. The placental and stem cell origin of NP-EVs supports their use in regenerative medicine, as recently proposed

545 (Mitrani et al., 2021).

546 Proteomic and miRNA profile analysis of amniotic fluid-EVs has been gaining an increasing 547 interest in identifying disease-associated biomarkers and mechanisms (Dixon et al., 2018). In 548 this study, we identified the specific increase in the levels of surface-associated CD105 in PE549 EVs, whereas other surface markers showed similar expression levels. Endoglin (CD105) is a co-receptor of TGF- $\beta 1$ and TGF- $\beta 3$, highly expressed on the surface of syncytiotrophoblasts and endothelial cells (Valluru et al., 2011). An increase in soluble endoglin is a hallmark of 
bioRxiv preprint doi: https://doi org/10.1101/2022.02 14 480331: this version posted February 14,2022 . The copyright holder for this preprint (which was not certified by peer review) is the author/funder, who has granted bioRxiv a license to display the preprint in perpetuity. It is made available under aCC-BY-NC-ND 4.0 International license.

552

preeclampsia (Mutter and Karumanchi, 2008; Schuster et al., 2020) and is responsible for endothelial damage by trapping soluble TGF- $\beta$ molecules (Valluru et al., 2011). We here confirmed the increased CD105 levels through different orthogonal techniques. The use of a CD105-based capture chip allowed us to demonstrate that the increase in CD105 expression was not due to HLA-G expressing placental EVs. The origin of CD105 expressing EVs could be possibly attributed to cells of fetal or fetal membrane origin in the fluid, including AFSCs. Indeed, the analysis of cultured amniotic fluid mesenchymal stromal cells showed that the released EVs were largely $\mathrm{CD} 105^{+}$, whereas the $\mathrm{HLA}-\mathrm{G}^{+} \mathrm{EVs}$, representing a minor fraction, did not co-express CD105. It would be of interest in future studies to evaluate the CD105/HLA$\mathrm{G}$ co-expression by cells isolated from preeclamptic amniotic fluid.

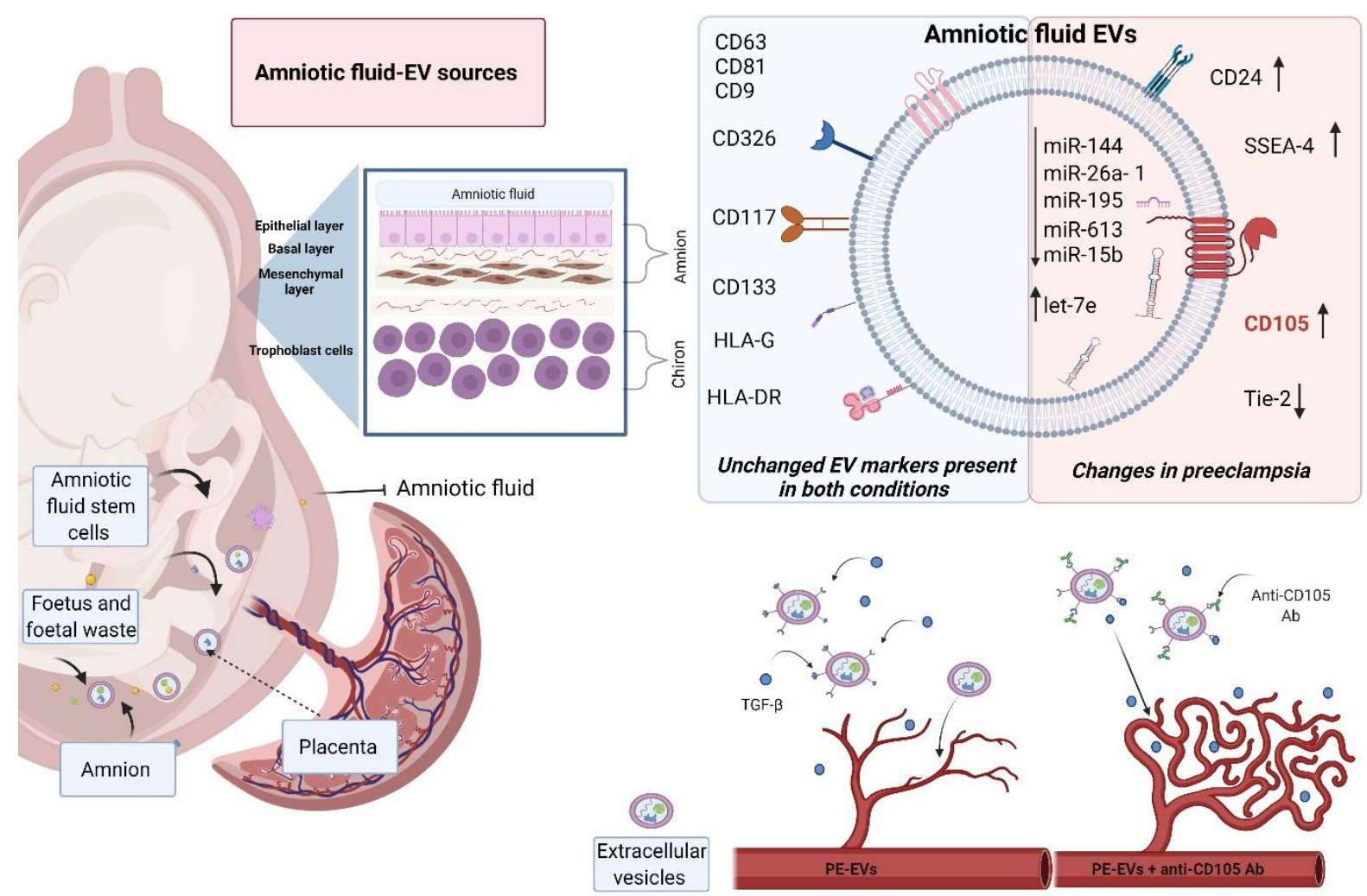

Figure 7. Graphical illustration summarizing the main findings. The figure shows the multiple possible sources of term amniotic fluid-derived EVs and the main changes in PE-EVs. Their antiangiogenic properties are supported by the specific upregulation of CD105 surface marker and the deregulation of several angiogenic miRNAs. The image was created with https://biorender.com. 
567 The increased CD105 level in PE-EVs is in agreement with previous data, showing higher

568 levels of endoglin on EVs produced by placental explants treated with preeclamptic sera

569 (Tannetta et al., 2013) and support the concept that surface receptors expressed by EVs may

570 act as a decoy for soluble factors (Tannetta et al., 2013; Sedrakyan et al., 2017). In analogy,

571 we found that PE-EVs affected angiogenesis in vitro and that this effect was abolished when

572 CD105 was blocked by a specific antibody. A slight antiangiogenic effect was also observed for NP-EVs, in parallel with data showing that AFSC-EVs do not promote angiogenesis despite their regenerative effect (Takov et al., 2020). However, this was not prevented by the antiCD105 antibody, suggesting the involvement of other factors. Previous data showed that administration of human EVs derived from preeclamptic placentas in mice resulted in damage to the vasculature, poor fetal nutrition and promoted endothelial permeability and glomeruli damage (Chang et al., 2018). In particular, the study provided an additional mechanism for the antiangiogenic effect of EVs, related to the presence of soluble endoglin transfer from the EV cargo to endothelial cells (Chang et al., 2018). Our results, showing the antiangiogenic effect of amniotic preeclamptic EVs, highlight a new role for EVs present in the amniotic fluid, possibly acting on both fetal and maternal compartments. Indeed, many reports described fetoplacental endothelial dysfunction in preeclampsia, as a detriment to both the mother and the fetus (Escudero et al., 2016). It would be of interest to investigate the possible effect of PEEVs on fetal development.

Several miRNAs with relevant roles in placenta development and angiogenesis have been

587 detected in biological fluids and tissues of preeclamptic patients, including specific miRNA 588 sets in the deriving EVs and are considered as a potential biomarker and "fingerprint" of placental dysfunction (Fallen et al., 2018; Gray et al.; 2017; Menon et al., 2019; Salomon et 
differential expression in 6 miRNAs, involved in trophoblast proliferation and invasion (let-7 and miR-613), as well as in the modulation of placental function (miR-15, miR-26a) and not overlap with the EV miRNA profile alterations described in maternal circulation (Fallen et al., 2018; Gray et al.; 2017; Menon et al., 2019; Salomon et al., 2017), the majority (let-7, miR-15b, miR-195, and miR-26a-1) have been reported to be modulated in placental tissue of preeclamptic patients (Cross et al., 2015; Mayor-Lynn et al., 2011; Hu et al., 2009; Bai et al., 2012; Choi et al., 2013). This underlines a differential profile of amniotic fluid-EVs from those in the maternal circulation, and may further support their origin from placental deriving cells.

602 These differentially expressed miRNAs may target genes involved in various functions, including development and angiogenesis, and are tightly involved in VEGF regulation and vascular remodeling. Indeed, deregulated pathways associated with angiogenesis, invasion and inflammation have been previously reported by target interaction analysis of miRNA content carried by preeclamptic maternal blood (Pillay et al., 2019, Salomon et al., 2017).

607 In conclusion, our study characterizes at single-EV level, EVs present in the amniotic fluid, an 608 interesting biofluid with a possible relevance for therapeutic application. Amniotic fluidderived EVs showed a heterogeneous origin, expressing markers of fetal and placental cells.

610 We highlight the differential expression of antiangiogenic factors in normal and preeclamptic 611 amniotic fluid-derived EVs, both at surface and cargo level. These characteristics may reflect 612 the hypoxic and antiangiogenic general status of preeclampsia and could possibly play a role 613 by affecting the developing fetus or the surrounding fetal membranes. Finally, EVs within the 614 amniotic fluid could potentially be used as a diagnostic test if investigated at earlier stages of 615 pregnancy. 
617 Acknowledgements:

618 We thank Dr. Sharad Kholia for his help with the figure and manuscript editing and correction.

619 We thank TRACON Pharmaceuticals (San Diego, CA) for providing the anti-CD105 Ab TRC-

620105.

621 Funding: The study was supported by the European Union's Horizon 2020 research and

622 innovation program under the Marie Skłodowska-Curie grant iPlacenta, agreement No.765274

623 and RenalToolBox No.813839. NG, JS, VG and RS are part of the iPLACENTA (NG, JS and

624 VG) and RenalToolBox (RS) projects.

625

626

627 
References:

Amaral, L. M. et al. (2015) 'Preeclampsia: Long-term consequences for vascular health', Vascular Health and Risk Management, 11, pp. 403-415. doi: 10.2147/VHRM.S64798.

Baghaei, K. et al. (2017) 'Isolation, differentiation, and characterization of mesenchymal stem cells from human bone marrow', Gastroenterology and Hepatology from Bed to Bench, 10(3), pp. 208-213. doi: 10.22037/ghfbb.v0i0.1089.

Bai, Y. et al. (2012) 'Downregulated miR-195 detected in Preeclamptic Placenta affects trophoblast cell invasion via modulating ActRIIA expression', PLoS ONE, 7(6). doi: 10.1371/journal.pone.0038875.

Balbi, C. et al. (2017) 'First characterization of human amniotic fluid stem cell extracellular vesicles as a powerful paracrine tool endowed with regenerative potential', Stem Cells Translational Medicine, 6(5), pp. 1340-1355. doi: 10.1002/sctm.16-0297.

Bindea, G. et al. (2009) 'ClueGO: A Cytoscape plugin to decipher functionally grouped gene ontology and pathway annotation networks', Bioinformatics, 25(8), pp. 1091-1093. doi: 10.1093/bioinformatics/btp101.

Brossa, A., Buono, L. and Bussolati, B. (2018) 'Effect of the monoclonal antibody TRC105 in combination with Sunitinib on renal tumor derived endothelial cells', Oncotarget, 9(32), pp. 22680-22692. doi: 10.18632/oncotarget.25206.

Brown, M. A. et al. (2018) 'Hypertensive disorders of pregnancy: ISSHP classification, diagnosis, and management recommendations for international practice', Hypertension, 72(1), pp. 24-43. doi: 10.1161/HYPERTENSIONAHA.117.10803.

Cananzi, M., Atala, A. and De Coppi, P. (2009) 'Stem cells derived from amniotic fluid: New potentials in regenerative medicine', Reproductive BioMedicine Online, 18, pp. 17-27. doi: 10.1016/S1472-6483(10)60111-3.

Chang, X. et al. (2018) 'Exosomes from women with preeclampsia induced vascular dysfunction by delivering sFLT (soluble fms-like tyrosine kinase)-1 and SENG (soluble endoglin) to endothelial cells', Hypertension, 72(6), pp. 1381-1390. doi: 10.1161/HYPERTENSIONAHA.118.11706.

Chen, E. Y. et al. (2013) 'Enrichr: Interactive and collaborative HTML5 gene list enrichment analysis tool', BMC Bioinformatics, 14. doi: 10.1186/1471-2105-14-128.

Choi, S. Y. et al. (2013) 'MicroRNA expression profiles in placenta with severe preeclampsia using a PNA-based microarray', Placenta, 34(9), pp. 799-804. doi: 10.1016/j.placenta.2013.06.006.

Cronqvist, T. et al. (2017) 'Syncytiotrophoblast derived extracellular vesicles transfer functional placental miRNAs to primary human endothelial cells', Scientific Reports, 7(1), pp. 1-14. doi: 10.1038/s41598-017-04468-0.

Cross, C. E. et al. (2015) 'Oxidative Stress Alters miRNA and Gene Expression Profiles in Villous First Trimester Trophoblasts', BioMed Research International, 2015. doi: 
667 Dimuccio, V.et al. (2014) 'Urinary CD133+ extracellular vesicles are decreased in kidney 668 transplanted patients with slow graft function and vascular damage', PLoS ONE, 9(8). doi: 669 10.1371/journal.pone.0104490.

670 Dixon, C. L. et al. (2018) 'Amniotic fluid exosome proteomic profile exhibits unique

671 pathways of term and preterm labor', Endocrinology, 159(5), pp. 2229-2240. doi:

672 10.1210/en.2018-00073.

673 Duley, L. (2009) 'The global impact of pre-eclampsia and eclampsia.', Seminars in

674 perinatology, 33(3), pp. 130-7. doi: 10.1053/j.semperi.2009.02.010.

675 Escudero, C. A. et al. (2016) 'Role of extracellular vesicles and microRNAs on dysfunctional 676 angiogenesis during preeclamptic pregnancies', Frontiers in Physiology, 7(MAR), pp. 1-17. 677 doi: 10.3389/fphys.2016.00098.

678 Eunsung Mouradian, M. M. (2008) 'Immunomodulatory molecules are released from the first 679 trimester and term placenta via exosomes', Bone, 23(1), pp. 1-7. doi:

680 10.1016/j.placenta.2012.10.005.Immunomodulatory.

681 Fallen, S. et al. (2018) 'Extracellular vesicle RNAs reflect placenta dysfunction and are a biomarker source for preterm labour', Journal of Cellular and Molecular Medicine, 22(5), pp. 2760-2773. doi: 10.1111/jcmm.13570.

684

Gebara, N. et al. (2021) 'Angiogenic properties of placenta-derived extracellular vesicles in normal pregnancy and in preeclampsia', International Journal of Molecular Sciences, 22(10). doi: 10.3390/ijms22105402.

687 Gray, C. et al. (2017) 'Maternal plasma miRNAs as biomarkers during mid-pregnancy to 688 predict later spontaneous preterm birth: A pilot study', Scientific Reports, 7(1), pp. 1-7. doi: 689 10.1038/s41598-017-00713-8.

690 Han, C. et al. (2021) 'Single-vesicle imaging and colocalization analysis for tetraspanin 691 profiling of individual extracellular vesicles', Journal of Extracellular Vesicles, 10(3). doi: 692 10.1002/jev2.12047.

693 Hromadnikova, I. et al. (2015) 'Cardiovascular and cerebrovascular disease associated 694 microRNAS are dysregulated in placental tissues affected with gestational hypertension, 695 preeclampsia and intrauterine growth restriction', PLOS ONE, 10(9), pp. 1-27. doi:

696 10.1371/journal.pone.0138383.

$697 \mathrm{Hu}$, Y. et al. (2009) 'Differential expression of microRNAs in the placentae of Chinese 698 patients with severe pre-eclampsia', Clinical Chemistry and Laboratory Medicine, 47(8), pp. 699 923-929. doi: 10.1515/CCLM.2009.228.

700 Huang, H. Y. et al. (2020) 'MiRTarBase 2020: Updates to the experimentally validated microRNA-target interaction database', Nucleic Acids Research, 48(D1), pp. D148-D154.

702 doi: 10.1093/nar/gkz896.

703 Iampietro, C. et al. (2020) 'Full-Term Human Amniotic Fluid Mesenchymal Stem Cells Show 704 Nephroprotective Effect In An Acute Kidney Injury Model', Journal of Stem Cells Research, 
Development \& Therapy, 6(3), pp. 1-8. doi: 10.24966/srdt-2060/100041.

Jankovičová, J. et al. (2020) 'Tetraspanins, more than markers of extracellular vesicles in reproduction', International Journal of Molecular Sciences, 21(20), pp. 1-30. doi: 10.3390/ijms21207568.

Jassal, B. et al. (2020) 'The reactome pathway knowledgebase', Nucleic Acids Research, 48(D1), pp. D498-D503. doi: 10.1093/nar/gkz1031.

Kaufmann, P., Black, S. and Huppertz, B. (2003) 'Endovascular trophoblast invasion: Implications for the pathogenesis of intrauterine growth retardation and preeclampsia', Biology of Reproduction, 69(1), pp. 1-7. doi: 10.1095/biolreprod.102.014977.

Keller, S. et al. (2007) 'CD24 is a marker of exosomes secreted into urine and amniotic fluid', Kidney International, 72(9), pp. 1095-1102. doi: 10.1038/sj.ki.5002486.

Lai, X. et al. (2012) 'Computational analysis of target hub gene repression regulated by multiple and cooperative miRNAs', Nucleic Acids Research, 40(18), pp. 8818-8834. doi: 10.1093/nar/gks657.

Maere, S., Heymans, K. and Kuiper, M. (2005) 'BiNGO: a Cytoscape plugin to assess overrepresentation of Gene Ontology categories in Biological Networks', Bioinformatics, 21(16), pp. 3448-3449. doi: 10.1093/bioinformatics/bti551.

Martin, A. et al. (2010) 'BisoGenet: A new tool for gene network building, visualization and analysis', BMC Bioinformatics, 11. doi: 10.1186/1471-2105-11-91.

Mayor-Lynn, K. et al. (2011) 'Expression profile of microRNAs and mRNAs in human placentas from pregnancies complicated by preeclampsia and preterm labor', Reproductive Sciences, 18(1), pp. 46-56. doi: 10.1177/1933719110374115.

McMaster, M. et al. (1998) 'HLA-G isoforms produced by placental cytotrophoblasts and found in amniotic fluid are due to unusual glycosylation.', Journal of immunology, 160(12), pp. 5922-8. Available at: http://www.ncbi.nlm.nih.gov/pubmed/9637505.

Menon, R. et al. (2019) 'Circulating exosomal miRNA profile during term and preterm birth pregnancies: A longitudinal study', Endocrinology, 160(2), pp. 249-275. doi: 10.1210/en.2018-00836.

Mitrani, M. I. et al. (2021) 'Case Report: Administration of Amniotic Fluid-Derived Nanoparticles in Three Severely Ill COVID-19 Patients', Frontiers in Medicine, 8(March), pp. 1-8. doi: 10.3389/fmed.2021.583842.

Monguió-Tortajada, M. et al. (2019) 'Extracellular vesicle isolation methods: rising impact of size-exclusion chromatography', Cellular and Molecular Life Sciences, 76(12), pp. 23692382. doi: 10.1007/s00018-019-03071-y.

Montis C., et al. (2017). 'Size distribution of extracellular vesicles by optical correlation techniques', Colloids Surf B Biointerfaces, 158:331-338. doi: 10.1016/j.colsurfb.2017.06.047.

Mutter, W. P. and Karumanchi, S. A. (2008) 'Molecular mechanisms of preeclampsia', Microvascular Research, 75(1), pp. 1-8. doi: 10.1016/j.mvr.2007.04.009. 
743 Nair, S. et al. (2021) 'Placenta-Derived Exosomes and Syncytiotrophoblast Microparticles and their Role in Human Reproduction: Immune Modulation for Pregnancy Success', Seminars in Immunopathology, 72(5), pp. 440-457. doi: 10.1111/aji.12311.

746

747

748

749

750

751

752

753

754

755

756

757

758

759

760

761

762

763

764

765

766

767

768

769

770

771

772

773

774

775

776

777

778

779

Nair, S. and Salomon, C. (2018) 'Extracellular vesicles and their immunomodulatory functions in pregnancy', Seminars in Immunopathology, 40(5), pp. 425-437. doi: 10.1007/s00281-018-0680-2.

Noisa, P. et al. (2012) 'Identification and Characterisation of the Early Differentiating Cells in Neural Differentiation of Human Embryonic Stem Cells', PLoS ONE, 7(5). doi: 10.1371/journal.pone.0037129.

Ogata, H. et al. (1999) 'KEGG: Kyoto encyclopedia of genes and genomes', Nucleic Acids Research, pp. 29-34. doi: 10.1093/nar/27.1.29.

Orozco, A. F. et al. (2009) 'Placental Release of Distinct DNA-associated Micro-particles into Maternal Circulation: Reflective of Gestation Time and Preeclampsia', Placenta, 30(10), pp. 891-897. doi: 10.1016/j.placenta.2009.06.012.

Ospina-Prieto, S. et al. (2016) 'MicroRNA-141 is upregulated in preeclamptic placentae and regulates trophoblast invasion and intercellular communication', Translational Research, 172(March), pp. 61-72. doi: 10.1016/j.trsl.2016.02.012.

Pillay, P. et al. (2019) 'Exosomal microRNA profiling in early and late onset preeclamptic pregnant women reflects pathophysiology', International Journal of Nanomedicine, 14, pp. 5637-5657. doi: 10.2147/IJN.S208865.

Roubelakis, M. G., Trohatou, O. and Anagnou, N. P. (2012) 'Amniotic fluid and amniotic membrane stem cells: Marker discovery', Stem Cells International. doi: $10.1155 / 2012 / 107836$.

Rouillard, A. D. et al. (2016) 'The harmonizome: a collection of processed datasets gathered to serve and mine knowledge about genes and proteins', Database : the journal of biological databases and curation. doi: 10.1093/database/baw100.

Salomon, C. et al. (2017) 'Placental exosomes as early biomarker of preeclampsia: Potential role of exosomalmicrornas across gestation', Journal of Clinical Endocrinology and Metabolism, 102(9), pp. 3182-3194. doi: 10.1210/jc.2017-00672.

Schuster, J. et al. (2020) 'Placental extracellular vesicles and pre-eclampsia', American Journal of Reproductive Immunology, pp. 0-3. doi: 10.1111/aji.13297.

Sedrakyan, S. et al. (2017) 'Amniotic fluid stem cell-derived vesicles protect from VEGFinduced endothelial damage', Scientific Reports, 7(1), pp. 1-12. doi: 10.1038/s41598-01717061-2.

Shannon, P. et al. (2003) 'Cytoscape: A software Environment for integrated models of biomolecular interaction networks', Genome Research, 13(11), pp. 2498-2504. doi: 10.1101/gr.1239303. 
780 Skovronova, R. et al. (2021) 'Surface marker expression in small and medium/large

781 mesenchymal stromal cell-derived extracellular vesicles in naïve or apoptotic condition using

782 orthogonal techniques', Cells, 10 (11)(2948). doi.org/10.3390/cells10112948.

783 Staff, A. C. et al. (2007) 'Circulating concentrations of soluble endoglin (CD105) in fetal and

784 maternal serum and in amniotic fluid in preeclampsia', American Journal of Obstetrics and

785 Gynecology, 197(2), pp. 176.e1-176.e6. doi: 10.1016/j.ajog.2007.03.036.

786 Takov, K. et al. (2020) 'Small extracellular vesicles secreted from human amniotic fluid

787 mesenchymal stromal cells possess cardioprotective and promigratory potential', Basic

788 Research in Cardiology, 115(3), pp. 1-22. doi: 10.1007/s00395-020-0785-3.

789 Tannetta, D. S. et al. (2013) 'Characterisation of Syncytiotrophoblast Vesicles in Normal

790 Pregnancy and Pre-Eclampsia: Expression of Flt-1 and Endoglin', PLoS ONE, 8(2), pp. 1-29.

791 doi: 10.1371/journal.pone.0056754.

792 Théry, C. et al. (2018) 'Minimal information for studies of extracellular vesicles 2018

793 (MISEV2018): a position statement of the International Society for Extracellular Vesicles

794 and update of the MISEV2014 guidelines', Journal of Extracellular Vesicles, 7(1). doi:

$79510.1080 / 20013078.2018 .1535750$.

796 Tong, Z. et al. (2019) 'TransmiR v2.0: An updated transcription factor-microRNA regulation 797 database', Nucleic Acids Research, 47(D1), pp. D253-D258. doi: 10.1093/nar/gky1023.

798 Underwood, M. A., Gilbert, W. M. and Sherman, M. P. (2005) 'Amniotic fluid: Not just fetal 799 urine anymore', Journal of Perinatology, 25(5), pp. 341-348. doi: 10.1038/sj.jp.7211290.

800 Valluru, M. et al. (2011) 'Transforming growth factor- $\beta$ and endoglin signaling orchestrate 801 wound healing', Frontiers in Physiology, 2 NOV, pp. 1-12. doi: 10.3389/fphys.2011.00089. 